\title{
A mean value estimate for real character sums
}

\author{
by
}

\section{R. Heath-Brown (Oxford)}

1. Introduction. There are a number of well known estimates for averages of Dirichlet polynomials. For example one has

$$
\int_{0}^{T}\left|\sum_{n \leq N} a_{n} n^{-i t}\right|^{2} d t \ll(T+N) \sum_{n \leq N}\left|a_{n}\right|^{2},
$$

for any complex numbers $a_{n}$. This is a weak consequence of Theorem 6.1 of Montgomery [6]. Similarly, for Dirichlet polynomials involving characters one has

$$
\sum_{\chi(\bmod q)}\left|\sum_{n \leq N} a_{n} \chi(n)\right|^{2} \ll(q+N) \sum_{n \leq N}\left|a_{n}\right|^{2}
$$

and

$$
\sum_{q \leq Q} \sum_{\chi(\bmod q)}^{*}\left|\sum_{n \leq N} a_{n} \chi(n)\right|^{2} \ll\left(Q^{2}+N\right) \sum_{n \leq N}\left|a_{n}\right|^{2},
$$

where $\sum^{*}$ indicates summation over primitive characters only. These last two bounds follow respectively from Theorem 6.2 of Montgomery [6] and from the large sieve in the form due to Gallagher [2], for example. In each case one may interpret the upper bound as being composed of two terms, the first of which reflects the long term average, and the second of which reflects the contribution of a single point where the Dirichlet polynomial is large. Thus for example one has

$$
\int_{0}^{T}\left|\sum_{n \leq N} a_{n} n^{-i t}\right|^{2} d t \sim T \sum_{n \leq N}\left|a_{n}\right|^{2},
$$

as $T \rightarrow \infty$, and

$$
\left|\sum_{n \leq N} a_{n} n^{-i t}\right|^{2} \leq N \sum_{n \leq N}\left|a_{n}\right|^{2}
$$


for any $t \in[0, T]$. In each of (1), (2) and (3) the "long term average" is $\sum_{n}\left|a_{n}\right|^{2}$, as in (4), while the contribution from a single point is given by Cauchy's inequality, as in (5).

These mean-value estimates have important consequences for averages of the Riemann Zeta-function $\zeta(s)$ and Dirichlet $L$-functions $L(s, \chi)$, as well as for zero-density theorems for these functions, as in Montgomery [6; Chapters 10 and 12].

The purpose of this paper is to give an analogous mean-value estimate for sums over real characters. A weak result of the type we have in mind is given by Elliott [1] as

$$
\sum_{p \leq M}\left|\sum_{n \leq N} a_{n}\left(\frac{n}{p}\right)\right|^{2} \ll M \sum_{n_{1} n_{2}=\square, 2 \square}\left|a_{n_{1}} a_{n_{2}}\right|+N \log N\left(\sum_{n \leq N}\left|a_{n}\right|\right)^{2},
$$

where $p$ runs over odd primes, and where the notation in the first sum on the right means that the sum is over pairs $n_{1}, n_{2} \leq N$ for which $n_{1} n_{2}$ is either a square or twice a square. The proof of this result is straightforward. Indeed, if we allow $m$ to run over all positive odd integers then on expanding we have

$$
\sum_{m \leq M}^{\text {odd }}\left|\sum_{n \leq N} a_{n}\left(\frac{n}{m}\right)\right|^{2}=\sum_{n_{1}, n_{2}} a_{n_{1}} \bar{a}_{n_{2}} \sum_{m \leq M}\left(\frac{n_{1} n_{2}}{m}\right) .
$$

Here and throughout the paper $\sum^{\text {odd }}$ will indicate summation restricted to an odd variable. Now $\chi(m)=\left(n_{1} n_{2} / m\right)$ is a Dirichlet character of conductor at most $4 n_{1} n_{2} \leq 4 N^{2}$, and is non-principal unless $n_{1} n_{2}$ is a square. In the remaining case the Pólya-Vinogradov inequality yields

$$
\sum_{m \leq M}\left(\frac{n_{1} n_{2}}{m}\right) \ll N \log N
$$

so that

$$
\sum_{m \leq M}^{\text {odd }}\left|\sum_{n \leq N} a_{n}\left(\frac{n}{m}\right)\right|^{2} \ll M \sum_{n_{1} n_{2}=\square}\left|a_{n_{1}} a_{n_{2}}\right|+N \log N\left(\sum_{n \leq N}\left|a_{n}\right|\right)^{2} .
$$

The above estimate clearly implies Elliott's result. If we choose $a_{n}=1$ for $n$ a square, and $a_{n}=0$ otherwise, then the left hand side will be of order $M N$. Thus it is impossible to replace the first term on the right by something like $M \sum\left|a_{n}\right|^{2}$, which would be of order $M N^{1 / 2}$ only. However, if we insist that the coefficients $a_{n}$ are supported on the square-free integers then the double sum reduces to $\sum\left|a_{n}\right|^{2}$. On applying Cauchy's inequality to the sum $\left(\sum\left|a_{n}\right|\right)^{2}$, our bound becomes 


$$
\sum_{m \leq M}^{\text {odd }}\left|\sum_{n \leq N} \mu^{2}(n) a_{n}\left(\frac{n}{m}\right)\right|^{2} \ll\left(M+N^{2} \log N\right) \sum_{n \leq N}\left|a_{n}\right|^{2} .
$$

This form of Elliott's estimate is more readily comparable with (1), (2) and (3). However, it is clearly imperfect, in as much as the term $N^{2} \log N$ appears to over-estimate the contribution arising from an individual value of $m$. Indeed, referring to the original form of Elliott's bound, Montgomery [6; Chapter 9] says: "This can be expected to be sharp only when $N$ is smaller than $M^{1 / 2}$; it would be nice to have a result like this when $N$ is as large as $M$." However, one cannot hope for such an improvement of (6), since when $a_{n}=1$ for all $n$, the contribution from square values of $m$ will be of order $M^{1 / 2} N \sum\left|a_{n}\right|^{2}$. With this in mind we restrict $m$ also to run over square-free values only, so that our problem becomes to improve on the estimate

$$
\sum_{m \leq M}^{*}\left|\sum_{n \leq N}^{*} a_{n}\left(\frac{n}{m}\right)\right|^{2} \ll\left(M+N^{2} \log N\right) \sum_{n \leq N}^{*}\left|a_{n}\right|^{2},
$$

where $\sum^{*}$ henceforth indicates restriction to positive odd square-free values.

We can now state our primary result.

Theorem 1. Let $M, N$ be positive integers, and let $a_{1}, \ldots, a_{n}$ be arbitrary complex numbers. Then

$$
\sum_{m \leq M}^{*}\left|\sum_{n \leq N}^{*} a_{n}\left(\frac{n}{m}\right)\right|^{2} \ll_{\varepsilon}(M N)^{\varepsilon}(M+N) \sum_{n \leq N}^{*}\left|a_{n}\right|^{2},
$$

for any $\varepsilon>0$.

From this one may easily deduce several corollaries. Firstly we extend our result to other primitive real characters, these being of the form $\chi(n)=$ $(n / m) \eta(n)$, where $\eta(n)$ is a primitive character to modulus 4 or 8 .

Corollary 1. Let $N, Q$ be positive integers, and let $a_{1}, \ldots, a_{n}$ be arbitrary complex numbers. Let $S(Q)$ denote the set of all real primitive characters of conductor at most $Q$. Then

$$
\sum_{\chi \in S(Q)}\left|\sum_{n \leq N} \mu^{2}(n) a_{n} \chi(n)\right|^{2} \ll_{\varepsilon}(Q N)^{\varepsilon}(Q+N) \sum_{n \leq N} \mu^{2}(n)\left|a_{n}\right|^{2},
$$

for any $\varepsilon>0$.

Our second corollary allows us to sum over values of $n$ that are not necessarily square-free. 
Corollary 2. Let $N, Q$ be positive integers, and let $a_{1}, \ldots, a_{n}$ be arbitrary complex numbers. Let $S(Q)$ be as in Corollary 1. Then

$$
\sum_{\chi \in S(Q)}\left|\sum_{n \leq N} a_{n} \chi(n)\right|^{2} \ll_{\varepsilon}(Q N)^{\varepsilon}(Q+N) \sum_{n_{1} n_{2}=\square}\left|a_{n_{1}} a_{n_{2}}\right|,
$$

for any $\varepsilon>0$.

Our next corollary simplifies the above result, at the expense of using the supremum norm for the $a_{n}$.

Corollary 3. Let $N, Q$ be positive integers, and let $a_{1}, \ldots, a_{n}$ be arbitrary complex numbers. Let $S(Q)$ be as in Corollary 1. Then

$$
\sum_{\chi \in S(Q)}\left|\sum_{n \leq N} a_{n} \chi(n)\right|^{2} \ll_{\varepsilon} Q^{\varepsilon} N^{1+\varepsilon}(Q+N) \max _{n \leq N}\left|a_{n}\right|^{2},
$$

for any $\varepsilon>0$.

We can also give an estimate for a bilinear form.

Corollary 4. Let $M, N$ be positive integers, and let $a_{1}, \ldots, a_{M}$ and $b_{1}, \ldots, b_{N}$ be arbitrary complex numbers satisfying $\left|a_{m}\right|,\left|b_{n}\right| \leq 1$. Then

$$
\sum_{m \leq M}^{\text {odd }} \sum_{n \leq N} a_{m} b_{n}\left(\frac{n}{m}\right) \ll_{\varepsilon}(M N)^{\varepsilon}\left(M N^{1 / 2}+M^{1 / 2} N\right),
$$

for any $\varepsilon>0$.

We can use our results to prove a new mean-value estimate for Dirichlet $L$-functions.

Theorem 2. Let $S(Q)$ be as in Corollary 1. Then

$$
\sum_{\chi \in S(Q)}|L(\sigma+i t, \chi)|^{4} \ll_{\varepsilon}\left\{Q+(Q(|t|+1))^{2-2 \sigma}\right\}\{Q(|t|+1)\}^{\varepsilon}
$$

for any fixed $\sigma \in[1 / 2,1]$ and any $\varepsilon>0$. Indeed, if $1 / 2<\sigma<1$, and $\delta>0$, we have

$$
\sum_{\chi \in S(Q)}|L(\sigma+i t, \chi)|^{4}=C Q+o(Q),
$$

uniformly for $|t| \leq Q^{(2 \sigma-1) /(2-2 \sigma)-\delta}$, for a certain constant $C=C(\sigma+i t)$, given explicitly by (33).

In contrast, we note that Jutila [3] has proved that

$$
\sum_{\chi \in S(Q)} \int_{-T}^{T}|L(1 / 2+i t, \chi)|^{2} d t \ll_{\varepsilon}(Q T)^{1+\varepsilon}
$$


for $T \geq 1$. (Actually, Jutila's result is somewhat stronger.) Our theorem is weaker in that it does not include an average over $t$, but is stronger in that it bounds a fourth power. The large sieve may be used to show that

$$
\begin{gathered}
\sum_{\chi \in D(Q)} \int_{-T}^{T}|L(1 / 2+i t, \chi)|^{4} d t \ll_{\varepsilon} Q^{2+\varepsilon} T^{1+\varepsilon}, \\
\sum_{\chi \in D(Q)} \int_{-T}^{T}|L(1 / 2+i t, \chi)|^{6} d t \ll_{\varepsilon} Q^{\varepsilon} T^{\varepsilon}\left(Q^{2} T+(Q T)^{3 / 2}\right),
\end{gathered}
$$

and

$$
\sum_{\chi \in D(Q)} \int_{-T}^{T}|L(1 / 2+i t, \chi)|^{8} d t \ll_{\varepsilon}(Q T)^{2+\varepsilon},
$$

where $D(Q)$ is the set of all primitive characters of conductor at most $Q$. These results are weaker with respect to $Q$ than the bound given by Theorem 2.

Our approach does not allow us to replace Theorem 2 by an asymptotic formula when $\sigma=1 / 2$. For the second moment in place of the fourth this is, however, possible. Jutila [5] has shown that

$$
\sum_{\chi \in S(Q)}|L(1 / 2, \chi)|^{2}=c Q \log ^{3} Q+O_{\varepsilon}\left(Q(\log Q)^{5 / 2+\varepsilon}\right),
$$

for an explicit constant $c>0$, and Guo (to appear) has obtained an asymptotic formula in which one saves a small power of $Q$.

By using (7) and related estimates Jutila [4] has shown that

$$
\sum_{\chi \in S(Q)} N(\sigma, T, \chi) \ll_{\varepsilon}(Q T)^{(7-6 \sigma) /(6-4 \sigma)+\varepsilon},
$$

where $N(\sigma, T, \chi)$ is the number of zeros of $L(s, \chi)$ in the rectangle

$$
\sigma \leq \Re(s) \leq 1, \quad|\Im(s)| \leq T,
$$

as usual. As a corollary of this result he shows that

$$
\sum_{\chi \in S(Q)}\left|\sum_{p \leq Q^{\gamma}} \chi(p)\right| \ll_{\varepsilon} Q^{h(\gamma)+\varepsilon}
$$

where

$$
h(\gamma)= \begin{cases}1+\frac{1}{2} \gamma, & \gamma \leq \frac{1}{2}, \\ \frac{3}{2}(1+\gamma)-(2 \gamma)^{1 / 2}, & \frac{1}{2} \leq \gamma \leq 2, \\ \frac{1}{2}+\gamma, & \gamma \geq 2\end{cases}
$$

This estimate is now an immediate consequence of Corollary 4. Indeed, one 
has the improved exponent

$$
h(\gamma)=\max \left(1+\frac{1}{2} \gamma, \frac{1}{2}+\gamma\right) .
$$

Theorems 1 and 2 enable us to establish a new zero-density estimate.

Theorem 3. Let $S(Q)$ be as in Corollary 1. Then

$$
\sum_{\chi \in S(Q)} N(\sigma, T, \chi) \ll_{\varepsilon}(Q T)^{\varepsilon} Q^{3(1-\sigma) /(2-\sigma)} T^{(3-2 \sigma) /(2-\sigma)}
$$

for any $\varepsilon>0$.

This estimate is sharper than Jutila's bound (8) in its $Q$-aspect, but weaker with respect to $T$. This is natural, since Theorem 3 is based on Theorem 1, in which the saving is in the $Q$-aspect only.

2. Proof of Theorem 1; the plan of attack. The basic sum which we shall study is

$$
\Sigma_{1}=\sum_{M<m \leq 2 M}^{*}\left|\sum_{N<n \leq 2 N} a_{n}\left(\frac{n}{m}\right)\right|^{2},
$$

in which the coefficients $a_{n}$ are supported on the odd square-free integers in $(N, 2 N]$. It is a straightforward matter to recover an estimate for the sum in Theorem 1 from a bound for sums of the form $\Sigma_{1}$. We observe that

$$
\Sigma_{1} \ll M N \sum_{N<n \leq 2 N}\left|a_{n}\right|^{2}
$$

by Cauchy's inequality, so that we may define

$$
\mathcal{B}(M, N)=\sup \frac{\Sigma_{1}}{\sum_{N<n \leq 2 N}\left|a_{n}\right|^{2}},
$$

where the supremum is over all admissible sets of complex numbers $a_{n}$ for which the denominator is non-vanishing. The argument above yields the trivial bound

$$
\mathcal{B}(M, N) \ll M N .
$$

Naturally we aim to prove that $\mathcal{B}(M, N) \ll(M N)^{\varepsilon}(M+N)$.

Our first result shows that $\mathcal{B}(M, N)$ is, essentially, symmetric.

Lemma 1. We have $\mathcal{B}(M, N) \leq 2 \mathcal{B}(N, M)$. Moreover, there exist coefficients $a_{n}^{\prime}$ with $\left|a_{n}\right|=\left|a_{n}^{\prime}\right|$ such that

$$
\sum_{M<m \leq 2 M}^{*}\left|\sum_{N<n \leq 2 N} a_{n}\left(\frac{n}{m}\right)\right|^{2} \leq 2 \sum_{M<m \leq 2 M}^{*}\left|\sum_{N<n \leq 2 N} a_{n}^{\prime}\left(\frac{m}{n}\right)\right|^{2} .
$$

We shall prove this in the next section. 
Lemma 1 shows that we may replace the Jacobi symbol $\left(\frac{n}{m}\right)$ by $\left(\frac{m}{n}\right)$ in the definition of $\Sigma_{1}$, at the expense of a factor 2 . We then introduce a weight $W(m / M)$, given by

$$
W(x)= \begin{cases}\exp \left(-\frac{1}{(2 x-1)(5-2 x)}\right) & \text { if } 1 / 2<x<5 / 2, \\ 0 & \text { otherwise }\end{cases}
$$

This function is infinitely differentiable for all $x$. We now see that

$$
\Sigma_{1} \ll \sum_{m=-\infty}^{\infty} W\left(\frac{m}{M}\right)\left|\sum_{N<n \leq 2 N} a_{n}\left(\frac{m}{n}\right)\right|^{2} .
$$

The condition that $m$ should be square-free is very difficult to handle, and it turns out to be easier to include certain extra values of $m$ into the sum. For any positive integer $m$ we shall write $s(m)$ for the largest square-free factor of $m$. We then have

$$
\Sigma_{1} \ll \sum_{m ; s(m)>K}^{\text {odd }} W\left(\frac{m}{M}\right)\left|\sum_{n} a_{n}\left(\frac{m}{n}\right)\right|^{2}
$$

for any $K \leq M / 2$. The plan is now to estimate the expression on the right as the sum over all odd $m$, less the sum over those odd $m$ which have a large square factor. The two sums thus produced are more readily handled, at least if $K$ is not too large. On the other hand, if $K$ is not too small it turns out that there is no serious loss incurred by including the extra terms in the transition from (10) to (11). Roughly speaking, the critical size for $K$ is of order $N^{2} M^{-1}$, when $N \leq M$. With the above discussion in mind we therefore define $\mathcal{B}(M, N, K)$ to be the supremum of

$$
\left\{\sum_{m ; s(m)>K}^{\text {odd }} W\left(\frac{m}{M}\right)\left|\sum_{n} a_{n}\left(\frac{m}{n}\right)\right|^{2}\right\} /\left\{\sum_{n}\left|a_{n}\right|^{2}\right\}
$$

taken over all admissible sequences $a_{n}$, so that $\mathcal{B}(M, N) \ll \mathcal{B}(M, N, K)$.

We may expand the sum on the left of (11) to obtain

$$
\Sigma_{1} \ll \sum_{n_{1}, n_{2}} a_{n_{1}} \bar{a}_{n_{2}} \sum_{m ; s(m)>K}^{\text {odd }} W\left(\frac{m}{M}\right)\left(\frac{m}{n_{1} n_{2}}\right) .
$$

If $n_{1}$ and $n_{2}$ have a common factor, the character

$$
\chi(m)=\left(\frac{m}{n_{1} n_{2}}\right)
$$

is imprimitive. This causes considerable technical difficulties, but it turns out that we may restrict our attention to the case in which $\left(n_{1}, n_{2}\right)$ is small. We therefore take $a_{n}$ to be a sequence of complex numbers supported on 
the odd square-free integers in $(N, 2 N]$, and we write

$$
\Sigma_{2}=\sum_{\left(n_{1}, n_{2}\right)=\Delta} a_{n_{1}} \bar{a}_{n_{2}} \sum_{m ; s(m)>K}^{\text {odd }} W\left(\frac{m}{M}\right)\left(\frac{m}{n_{1} n_{2}}\right) .
$$

We then define $\mathcal{C}(M, N, K, \Delta)$ to be the supremum of

$$
\Sigma_{2} / \sum\left|a_{n}\right|^{2}
$$

taken over all admissible sequences $a_{n}$, and we proceed to investigate the norm $\mathcal{C}(M, N, K, \Delta)$ for small values of $\Delta$. It is quite easy to bound $\mathcal{B}(M, N, K)$ in terms of $\mathcal{C}(M, N, K, \Delta)$, as the following lemma shows.

Lemma 2. Let $\varepsilon>0$ be given. Then for any positive number $K \leq M / 2$ and any $\Delta_{0}$ in the range $1 \leq \Delta_{0} \leq N$ we have

$$
\mathcal{B}(M, N, K) \ll N^{\varepsilon} \mathcal{B}\left(M, N_{1}, K\right)+\sum_{\Delta \leq \Delta_{0}} \mathcal{C}(M, N, K, \Delta),
$$

for some $N_{1} \leq N / \Delta_{0}$.

This will be proved in Section 4 .

To handle $\mathcal{C}(M, N, K, \Delta)$ we decompose $\Sigma_{2}$ as $\Sigma_{3}-\Sigma_{4}$ with

$$
\Sigma_{3}=\sum_{\left(n_{1}, n_{2}\right)=\Delta} a_{n_{1}} \bar{a}_{n_{2}} \sum_{m}^{\text {odd }} W\left(\frac{m}{M}\right)\left(\frac{m}{n_{1} n_{2}}\right)
$$

and

$$
\Sigma_{4}=\sum_{\left(n_{1}, n_{2}\right)=\Delta} a_{n_{1}} \bar{a}_{n_{2}} \sum_{m ; s(m) \leq K}^{\text {odd }} W\left(\frac{m}{M}\right)\left(\frac{m}{n_{1} n_{2}}\right) .
$$

Our analysis of these sums produces a main term for each, together with a number of subsidiary terms, all of which will be estimated via the norm $\mathcal{B}(M, N)$. It turns out that the two leading terms cancel to a large extent. Specifically, we prove in Section 5 the following.

Lemma 3. Let $\varepsilon>0$ be given. Let $N>\Delta$ and

$$
N^{2} M^{-1}(M N)^{\varepsilon} \leq K \leq M(M N)^{-\varepsilon} .
$$

For any two positive integers $n_{1}, n_{2}$ we write

$$
q=q\left(n_{1}, n_{2}\right)=\frac{n_{1} n_{2}}{\left(n_{1}, n_{2}\right)^{2}} .
$$

Moreover, we define

$$
\kappa(\Delta, q)=\prod_{p \mid \Delta}\left(1-\left(\frac{p}{q}\right) p^{-1 / 2}\right)
$$


and

Then

$$
m_{0}=\min \left\{1, \frac{N / \sqrt{M B}}{D_{1} D_{2}}\right\}
$$

where

$$
\Sigma_{3}=M_{3}+E_{3} \sum\left|a_{n}\right|^{2}
$$

$$
M_{3}=\sum_{b \leq K}^{*} \sqrt{\frac{M}{b}} \sum_{\left(n_{1}, n_{2}\right)=\Delta} a_{n_{1}} \bar{a}_{n_{2}} \frac{\phi(q)}{2 q} \kappa(\Delta, q)\left(\frac{b}{q}\right) \int_{0}^{\infty} W\left(x^{2}\right) d x,
$$

and

$$
E_{3} \ll_{\varepsilon} 1+(M N)^{\varepsilon} \Delta \frac{M}{N} m_{0} \sqrt{D_{1} D_{2}} \mathcal{B}\left(B, \frac{N}{D_{1} \Delta}\right)^{1 / 2} \mathcal{B}\left(B, \frac{N}{D_{2} \Delta}\right)^{1 / 2}
$$

for certain values of $B, D_{1}$ and $D_{2}$, subject to $D_{1}, D_{2} \gg(M N)^{-\varepsilon}$, and $1 \ll B \ll K$.

Similarly, in Section 6 we shall prove:

Lemma 4. Let $N>\Delta$ and $0<K \leq M^{1-\varepsilon}$. Then

$$
\Sigma_{4}=M_{4}+E_{4} \sum\left|a_{n}\right|^{2}
$$

where

$$
M_{4}=\sum_{\substack{v \leq K \\(v, 2 \Delta)=1}}^{*} \sqrt{\frac{M}{v}} \sum_{\left(n_{1}, n_{2}\right)=\Delta} a_{n_{1}} \bar{a}_{n_{2}} \frac{\phi(q \Delta)}{2 q \Delta}\left(\frac{v}{q}\right) \int_{0}^{\infty} W\left(x^{2}\right) d x
$$

and

$$
E_{4} \ll_{\varepsilon} 1+(M N)^{\varepsilon}\left(\frac{M}{B D_{1} D_{2}}\right)^{1 / 2} \mathcal{B}\left(B, \frac{N}{D_{1} \Delta}\right)^{1 / 2} \mathcal{B}\left(B, \frac{N}{D_{2} \Delta}\right)^{1 / 2},
$$

for certain values of $B, D_{1}, D_{2} \gg(M N)^{-\varepsilon}$, subject to $B \ll K$ and

$$
D_{1} D_{2} \gg(M N)^{-\varepsilon} \Delta^{-1} M^{1 / 2} B^{-1 / 2} \text {. }
$$

The next lemma, which we prove in Section 7 , demonstrates that the leading terms in Lemmas 3 and 4 are indeed very similar.

LemMA 5. For $1 \ll \Delta \ll N$ and $0<K \leq M$ we have

$$
M_{3}-M_{4} \ll M^{1 / 2} K^{-1 / 2}(M N)^{\varepsilon} \mathcal{B}\left(K \Delta^{2}(M N)^{\varepsilon}, N(M N)^{\varepsilon}\right) .
$$

The strategy of the proof is now to combine Lemmas $2-5$ to provide a recursive estimate for $\mathcal{B}(M, N)$. We begin, in Section 8, by using Lemmas $3-5$ to bound $\mathcal{C}(M, N, K, \Delta)$ in terms of $\mathcal{B}$.

Lemma 6. Suppose $1<\xi \leq 2$ is such that

$$
\mathcal{B}(M, N) \ll_{\varepsilon}(M N)^{\varepsilon}\left(M+N^{\xi}\right)
$$


for any $\varepsilon>0$ and $1 \leq N \leq M$. Then

$$
\mathcal{C}(M, N, K, \Delta) \ll \Delta^{4}(M N)^{\varepsilon}\left(M+N+M^{1 / 2} K^{\xi-1 / 2}+M^{1 / 2} N K^{-1 / 2}\right),
$$

providing that $1 \leq \Delta<N$ and $N^{2} M^{-1}(M N)^{\varepsilon}<K \leq M(M N)^{-\varepsilon}$.

In view of the estimate (6), established in our introduction, we see that $\xi=2$ is admissible here.

When the above result is inserted into Lemma 2 one deduces a new bound for $\mathcal{B}(M, N)$. The outcome, in Section 8 , is as follows.

LEMMA 7. If (14) holds for any $\varepsilon>0$, then

$$
\mathcal{B}(M, N) \ll_{\varepsilon}(M N)^{\varepsilon}\left(M+M^{1-\xi} N^{(2 \xi-1)}\right)
$$

for any $\varepsilon>0$.

Finally, we deduce a new bound of the form (14), again in Section 8.

LEMma 8. If (14) holds for any $\varepsilon>0$, with $\xi>1$, then

$$
\mathcal{B}(M, N) \ll_{\varepsilon}(M N)^{\varepsilon}\left(M+N^{(2 \xi-1) / \xi}\right)
$$

for any $\varepsilon>0$.

Since $(2 \xi-1) / \xi<\xi$ for $\xi>1$ we see that the infimum of the possible values for $\xi$ must be 1 , and Theorem 1 follows.

3. Preliminary lemmas. In this section we shall prove various results which are either of independent interest, or which will be required more than once in the proof of Theorem 1.

We begin by establishing Lemma 1 , given in the previous section. To do this we use the duality principle, as given by Montgomery [6; Chapter 9]. This shows that $\mathcal{B}(M, N)$ is the supremum of

$$
\left\{\sum_{N<n \leq 2 N}^{*}\left|\sum_{M<m \leq 2 M}^{*} b_{m}\left(\frac{n}{m}\right)\right|^{2}\right\} /\left\{\sum_{M<m \leq 2 M}^{*}\left|b_{m}\right|^{2}\right\},
$$

for complex numbers $b_{m}$ for which the denominator is non-zero. However, the law of quadratic reciprocity shows that if $n \equiv 1(\bmod 4)$ then

$$
\sum_{M<m \leq 2 M}^{*} b_{m}\left(\frac{n}{m}\right)=\sum_{M<m \leq 2 M}^{*} b_{m}\left(\frac{m}{n}\right),
$$

while if $n \equiv 3(\bmod 4)$ then

$$
\sum_{M<m \leq 2 M}^{*} b_{m}\left(\frac{n}{m}\right)=\sum_{M<m \leq 2 M}^{*}(-1)^{(m-1) / 2} b_{m}\left(\frac{m}{n}\right) .
$$


We deduce that

$$
\begin{aligned}
\sum_{N<n \leq 2 N}^{*}\left|\sum_{M<m \leq 2 M}^{*} b_{m}\left(\frac{n}{m}\right)\right|^{2} & \leq \sum_{N<n \leq 2 N}^{*}\left|\sum_{M<m \leq 2 M}^{*} b_{m}\left(\frac{m}{n}\right)\right|^{2} \\
& +\sum_{N<n \leq 2 N}^{*}\left|\sum_{M<m \leq 2 M}^{*}(-1)^{(m-1) / 2} b_{m}\left(\frac{m}{n}\right)\right|^{2} \\
\leq & 2 \mathcal{B}(N, M) \sum_{M<m \leq 2 M}^{*}\left|b_{m}\right|^{2},
\end{aligned}
$$

and the first part of the lemma follows. For the second part we split the sum into two parts depending on the value of $m(\bmod 4)$ and take $a_{n}^{\prime}=a_{n}$ or $(-1)^{(n-1) / 2} a_{n}$ accordingly.

We remark that the argument here allows us to replace $\left(\frac{n}{m}\right)$ by $\left(\frac{m}{n}\right)$ in the definition of $\mathcal{B}(M, N)$, at the expense of a factor 2. We shall use this fact frequently in what follows, without further comment.

Our next result shows that $\mathcal{B}(M, N)$ is, essentially, increasing with respect to $M$ and $N$.

Lemma 9. There is an absolute constant $C \geq 1$ as follows. Let $M_{1}, N \geq$ 1 and $M_{2} \geq C M_{1} \log \left(2 M_{1} N\right)$. Then

$$
\mathcal{B}\left(M_{1}, N\right) \ll \mathcal{B}\left(M_{2}, N\right) .
$$

Similarly, if $M, N_{1} \geq 1$ and $N_{2} \geq C N_{1} \log \left(2 N_{1} M\right)$, then

$$
\mathcal{B}\left(M, N_{1}\right) \ll \mathcal{B}\left(M, N_{2}\right) .
$$

For the proof it is convenient to write $K=M_{2} / M_{1}$. We begin by considering an odd prime $p$ satisfying $2 K / 3<p<4 K / 3$. We observe that

$$
\left|\sum_{N<n \leq 2 N}^{*} a_{n}\left(\frac{n}{m}\right)\right|^{2} \leq 2\left|\sum_{\substack{N<n \leq 2 N \\ p \nmid n}}^{*} a_{n}\left(\frac{n}{m}\right)\right|^{2}+2\left|\sum_{\substack{N<n \leq 2 N \\ p \mid n}}^{*} a_{n}\left(\frac{n}{m}\right)\right|^{2},
$$

so that

$$
\sum_{m}\left|\sum_{n}\right|^{2} \leq \sum_{p \mid m}\left|\sum_{n}\right|^{2}+2 \sum_{p \nmid m}\left|\sum_{p \nmid n}\right|^{2}+2 \sum_{p \nmid m}\left|\sum_{p \mid n}\right|^{2} .
$$

We shall consider two cases depending on whether $m$ lies in $\left(M_{1}, \frac{3}{2} M_{1}\right]$ or $\left(\frac{3}{2} M_{1}, 2 M_{1}\right]$, and we shall restrict $p$ to the intervals $\left(K, \frac{4}{3} K\right)$ and $\left(\frac{2}{3} K, K\right)$ accordingly. For the second sum on the right we then have 


$$
\sum_{\substack{N<n \leq 2 N \\ p \nmid n}}^{*} a_{n}\left(\frac{n}{m}\right)=\sum_{\substack{N<n \leq 2 N \\ p \nmid n}}^{*} b_{n}\left(\frac{n}{p m}\right),
$$

where $b_{n}=\left(\frac{n}{p}\right) a_{n}$. Here $p m$ will be odd and square-free, and will lie in the interval $\left(M_{2}, 2 M_{2}\right]$, by our condition on $p$. It follows that

$$
\begin{aligned}
\sum_{\substack{\alpha M_{1}<m \leq \beta M_{1} \\
p \nmid m}}^{*}\left|\sum_{\substack{N<n \leq 2 N \\
p \nmid n}}^{*} a_{n}\left(\frac{n}{m}\right)\right|^{2} & \leq \sum_{M_{2}<r \leq 2 M_{2}}\left|\sum_{N<n \leq 2 N}^{*} b_{n}\left(\frac{n}{r}\right)\right|^{2} \\
& \leq \mathcal{B}\left(M_{2}, N\right) \sum_{n}\left|a_{n}\right|^{2},
\end{aligned}
$$

where $(\alpha, \beta)$ is either $(1,3 / 2)$ or $(3 / 2,2)$.

For the sum

$$
\sum_{p \nmid m}\left|\sum_{p \mid n}\right|^{2}
$$

we have

$$
\sum_{\substack{M_{1}<m \leq 2 M_{1} \\ p \nmid m}}^{*}\left|\sum_{\substack{N<n \leq 2 N \\ p \mid n}}^{*} a_{n}\left(\frac{n}{m}\right)\right|^{2} \leq \mathcal{B}\left(M_{1}, N\right) \sum_{p \mid n}\left|a_{n}\right|^{2} .
$$

We therefore see that

$$
\begin{aligned}
\sum_{\alpha M_{1}<m \leq \beta M_{1}}^{*}\left|\sum_{N<n \leq 2 N}^{*} a_{n}\left(\frac{n}{m}\right)\right|^{2} & \\
\ll & \sum_{\substack{M_{1}<m \leq 2 M_{1} \\
p \mid m}}^{*}\left|\sum_{N<n \leq 2 N}^{*} a_{n}\left(\frac{n}{m}\right)\right|^{2} \\
& +\mathcal{B}\left(M_{2}, N\right) \sum_{n}\left|a_{n}\right|^{2}+\mathcal{B}\left(M_{1}, N\right) \sum_{p \mid n}\left|a_{n}\right|^{2}
\end{aligned}
$$

with $\alpha$ and $\beta$ as before, and $p$ in the appropriate range.

We now sum over all relevent primes $p$. We note that the number of available primes is of exact order $K / \log K$, and that the integers $m, n$ can have $O\left(\log \left(2 M_{1} N\right) / \log K\right)$ prime factors from the interval under consideration. We deduce that

$$
\begin{aligned}
& \frac{K}{\log K} \sum_{\alpha M_{1}<m \leq \beta M_{1}}^{*}\left|\sum_{N<n \leq 2 N}^{*} a_{n}\left(\frac{n}{m}\right)\right|^{2} \\
& \ll \frac{\log \left(2 M_{1} N\right)}{\log K} \sum_{M_{1}<m \leq 2 M_{1}}^{*}\left|\sum_{N<n \leq 2 N}^{*} a_{n}\left(\frac{n}{m}\right)\right|^{2}
\end{aligned}
$$




$$
\begin{aligned}
& +\frac{K}{\log K} \mathcal{B}\left(M_{2}, N\right) \sum_{n}\left|a_{n}\right|^{2}+\frac{\log \left(2 M_{1} N\right)}{\log K} \mathcal{B}\left(M_{1}, N\right) \sum_{n}\left|a_{n}\right|^{2} \\
\ll & \left\{\frac{\log \left(2 M_{1} N\right)}{\log K} \mathcal{B}\left(M_{1}, N\right)+\frac{K}{\log K} \mathcal{B}\left(M_{2}, N\right)\right. \\
& \left.+\frac{\log \left(2 M_{1} N\right)}{\log K} \mathcal{B}\left(M_{1}, N\right)\right\} \sum_{n}\left|a_{n}\right|^{2} .
\end{aligned}
$$

On combining the alternative ranges for $m$ this yields

$$
\begin{aligned}
\frac{K}{\log K} \sum_{M_{1}<m \leq 2 M_{1}}^{*}\left|\sum_{N<n \leq 2 N}^{*} a_{n}\left(\frac{n}{m}\right)\right|^{2} \\
\quad \ll\left\{\frac{\log \left(2 M_{1} N\right)}{\log K} \mathcal{B}\left(M_{1}, N\right)+\frac{K}{\log K} \mathcal{B}\left(M_{2}, N\right)\right\} \sum_{n}\left|a_{n}\right|^{2} .
\end{aligned}
$$

We may choose the coefficients $a_{n}$ so that

$$
\sum_{M_{1}<m \leq 2 M_{1}}^{*}\left|\sum_{N<n \leq 2 N}^{*} a_{n}\left(\frac{n}{m}\right)\right|^{2}=\mathcal{B}\left(M_{1}, N\right) \sum_{n}\left|a_{n}\right|^{2} .
$$

Thus if $K \geq C \log \left(2 M_{1} N\right)$ with a sufficiently large absolute constant $C$, we will deduce that

$$
\mathcal{B}\left(M_{1}, N\right) \ll \mathcal{B}\left(M_{2}, N\right),
$$

as claimed. Of course we may deduce the second part of the lemma from the first, via Lemma 1.

The next result allows us to estimate sums involving two different sequences, and with restrictions on the indices, in terms of the norm $\mathcal{B}$.

Lemma 10. Let $a_{n}, b_{n}$ be sequences of complex numbers supported on the odd square-free integers in $(N, 2 N]$. Then there exist $D_{1}, D_{2}$, satisfying

$$
\frac{1}{\log (2 M N)} \ll D_{i} \ll D \quad \text { and } \quad \frac{D}{\log ^{2}(2 M N)} \ll D_{1} D_{2} \ll \frac{D}{\log ^{2}(2 M N)},
$$

such that

$$
\begin{aligned}
& \sum_{D<d \leq 2 D} \sum_{M<m \leq 2 M}^{*}\left|\sum_{\substack{\left(n_{1}, n_{2}\right)=1 \\
d \mid n_{1} n_{2}}} a_{n_{1}} b_{n_{2}}\left(\frac{m}{n_{1} n_{2}}\right)\right| \\
& \ll(M N)^{\eta}\left(D_{1} D_{2}\right)^{1 / 2}\left\{\mathcal{B}\left(M, N / D_{1}\right) \sum\left|a_{n}\right|^{2}\right\}^{1 / 2}\left\{\mathcal{B}\left(M, N / D_{2}\right) \sum\left|b_{n}\right|^{2}\right\}^{1 / 2}, \\
& \text { for any } \eta>0 .
\end{aligned}
$$


If we decompose the sum over $n_{1}, n_{2}$ according to the value of $\left(n_{1}, d\right)=$ $d_{1}$, say, we find that

$$
\sum_{d}\left|\sum_{\substack{\left(n_{1}, n_{2}\right)=1 \\ d \mid n_{1} n_{2}}}\right| \leq \sum_{d} \sum_{d_{1} \mid d}\left|\sum_{n_{1}, n_{2}}\right|
$$

where the final sum is subject to the condition $\left(n_{1}, n_{2}\right)=1$ together with the requirements that $\left(n_{1}, d\right)=d_{1}$ and $d d_{1}^{-1} \mid n_{2}$. If we now write $d_{2}=d / d_{1}$ it follows that the sum, $S$ say, in Lemma 10 satisfies

$$
S \leq \sum_{D<d_{1} d_{2} \leq 2 D} \sum_{M<m \leq 2 M}^{*}\left|\sum_{\substack{\left(n_{1}, n_{2}\right)=1 \\ d_{1}\left|n_{1}, d_{2}\right| n_{2}}} a_{n_{1}} b_{n_{2}}\left(\frac{m}{n_{1} n_{2}}\right)\right| .
$$

We now pick out the condition $\left(n_{1}, n_{2}\right)=1$ by introducing a factor

$$
\sum_{d \mid n_{1}, n_{2}} \mu(d) \text {. }
$$

We therefore see that

$$
S \leq \sum_{d \leq 2 N} \sum_{D<d_{1} d_{2} \leq 2 D} \sum_{M<m \leq 2 M}^{*}\left|\sum_{d d_{1}\left|n_{1}, d d_{2}\right| n_{2}} a_{n_{1}} b_{n_{2}}\left(\frac{m}{n_{1} n_{2}}\right)\right| .
$$

At this point we decompose the available ranges for $d_{1}$ and $d_{2}$ into intervals $D_{i}<d_{i} \leq 2 D_{i}$, where each $D_{i}$ runs over powers of 2 and $D \ll D_{1} D_{2} \ll D$. Since there are $O(\log D)$ possible pairs $D_{1}, D_{2}$, and the lemma is trivial unless $D \ll N$, we see that there is some pair $D_{1}, D_{2}$ for which

$$
S \ll(\log N) \sum_{d \leq 2 N} \sum_{D_{i}<d_{i} \leq 2 D_{i}} \sum_{M<m \leq 2 M}^{*}\left|\sum_{d d_{1}\left|n_{1}, d d_{2}\right| n_{2}} a_{n_{1}} b_{n_{2}}\left(\frac{m}{n_{1} n_{2}}\right)\right| .
$$

The double sum over $n_{1}, n_{2}$ factorizes as

$$
\left\{\sum_{d d_{1} \mid n_{1}} a_{n_{1}}\left(\frac{m}{n_{1}}\right)\right\}\left\{\sum_{d d_{2} \mid n_{2}} b_{n_{2}}\left(\frac{m}{n_{2}}\right)\right\} .
$$

Hence Cauchy's inequality shows that

$$
S \ll(\log N) \Sigma_{a}^{1 / 2} \Sigma_{b}^{1 / 2},
$$

where

$$
\Sigma_{a}=\sum_{d \leq 2 N} \sum_{D_{i}<d_{i} \leq 2 D_{i}} \sum_{M<m \leq 2 M}^{*}\left|\sum_{d d_{1} \mid n} a_{n}\left(\frac{m}{n}\right)\right|^{2},
$$

and similarly for $\Sigma_{b}$. We now put $a_{n, d_{1}}=a_{n / d_{1}}$ when $d_{1} \mid n$, and $a_{n, d_{1}}=0$ 
otherwise. Then

$$
\left|\sum_{d d_{1} \mid n} a_{n}\left(\frac{m}{n}\right)\right| \leq\left|\sum_{d \mid n} a_{n, d_{1}}\left(\frac{m}{n}\right)\right|
$$

Moreover,

$$
\begin{aligned}
\sum_{M<m \leq 2 M}^{*}\left|\sum_{d \mid n} a_{n, d_{1}}\left(\frac{m}{n}\right)\right|^{2} & \leq \mathcal{B}\left(M, N / d_{1}\right) \sum_{d \mid n}\left|a_{n, d_{1}}\right|^{2} \\
& =\mathcal{B}\left(M, N / d_{1}\right) \sum_{d d_{1} \mid n}\left|a_{n}\right|^{2} .
\end{aligned}
$$

However, Lemma 9 yields $\mathcal{B}\left(M, N / d_{1}\right) \ll \mathcal{B}\left(M, \theta N / D_{1}\right)$ for $\theta=C \log (2 M N)$, and so

$$
\begin{aligned}
\Sigma_{a} & \ll \sum_{d \leq 2 N} \sum_{D_{i}<d_{i} \leq 2 D_{i}} \mathcal{B}\left(M, \theta N / D_{1}\right) \sum_{d d_{1} \mid n}\left|a_{n}\right|^{2} \\
& \ll N^{\eta / 2} D_{2} \mathcal{B}\left(M, \theta N / D_{1}\right) \sum_{n}\left|a_{n}\right|^{2},
\end{aligned}
$$

and similarly for $\Sigma_{b}$. Since $D \ll D_{1} D_{2} \ll D$, the lemma now follows, on replacing $D_{i}$ by $\theta D_{i}$.

Our fourth result applies the Poisson summation formula to a weighted character sum.

LEMma 11. Let $W(x)$ be an infinitely differentiable function supported on a compact subinterval of $(0, \infty)$. Then if $q$ is odd, positive and square-free we have

$$
\sum_{m=1}^{\infty} W\left(\frac{m}{M}\right)\left(\frac{m}{q}\right)=\frac{M \tau(q)}{q} \sum_{h=-\infty}^{\infty} \widehat{W}\left(\frac{h}{q / M}\right)\left(\frac{h}{q}\right),
$$

where $\tau(q)$ is the usual quadratic Gauss sum.

For the proof we split the sum on the left into residue classes, and apply the Poisson summation formula to each. This yields

$$
\begin{aligned}
\sum_{m=1}^{\infty} W\left(\frac{m}{M}\right)\left(\frac{m}{q}\right) & =\sum_{a(\bmod q)}\left(\frac{a}{q}\right) \sum_{b=-\infty}^{\infty} W\left(\frac{a+b q}{M}\right) \\
& =\sum_{a(\bmod q)}\left(\frac{a}{q}\right) \sum_{h=-\infty}^{\infty} \int_{-\infty}^{\infty} W\left(\frac{a+x q}{M}\right) e(-h x) d x \\
& =\sum_{a(\bmod q)}\left(\frac{a}{q}\right) \sum_{h=-\infty}^{\infty} e_{q}(a h) \frac{M}{q} \widehat{W}\left(\frac{h}{q / M}\right) .
\end{aligned}
$$


The lemma now follows from the relation

$$
\sum_{a(\bmod q)}\left(\frac{a}{q}\right) e_{q}(a h)=\tau(q)\left(\frac{h}{q}\right),
$$

which holds for odd square-free $q$.

Our next result will be used to "separate the variables" in a function of a product.

LEMma 12. Let $\varrho: \mathbb{R} \rightarrow \mathbb{R}$ be an infinitely differentiable function whose derivatives satisfy $\varrho^{(k)}(x) \ll_{k, A}|x|^{-A}$ for $|x| \geq 1$, for any positive constant A. Let

$$
\varrho_{+}(s)=\int_{0}^{\infty} \varrho(x) x^{s-1} d x
$$

and

$$
\varrho_{-}(s)=\int_{0}^{\infty} \varrho(-x) x^{s-1} d x .
$$

Then $\varrho_{+}(s)$ and $\varrho_{-}(s)$ are holomorphic in $\Re(s)=\sigma>0$, and satisfy

$$
\varrho_{+}(s), \varrho_{-}(s) \ll_{A, \sigma}|s|^{-A}
$$

there, for any positive constant A. Moreover, if $\sigma>0$ we have

$$
\varrho(x)=\frac{1}{2 \pi i} \int_{\sigma-i \infty}^{\sigma+i \infty} \varrho_{+}(s) x^{-s} d s
$$

and

$$
\varrho(-x)=\frac{1}{2 \pi i} \int_{\sigma-i \infty}^{\sigma+i \infty} \varrho_{-}(s) x^{-s} d s
$$

for any positive $x$.

This requires little comment. The bounds for $\varrho_{ \pm}(s)$ may be obtained by repeated integration by parts, and the expressions for $\varrho( \pm x)$ are examples of the Mellin inversion formula.

Our next result is an attempt to carry out the Poisson summation formula over the integers coprime to a given natural number $k$.

LEMma 13. Let $\psi: \mathbb{R} \rightarrow \mathbb{R}$ be an infinitely differentiable function with derivatives satisfying $\psi^{(j)}(x) \ll_{j, A}|x|^{-A}$ for $|x|>0$, for any positive constant $A$. Let $0<X_{1} \leq X \leq X_{2}$, and let $0<J \leq \min \left(X / X_{1}, X_{2} / X\right)$. Then for any integer $k \geq 2$ we have 


$$
\begin{aligned}
\sum_{(n, k)=1} \psi\left(\frac{n}{X}\right)= & \frac{\phi(k)}{k} X \int_{-\infty}^{\infty} \psi(x) d x-\psi(0) \sum_{d \mid k, d \leq X_{2}} \mu(d) \\
& -\sum_{d \mid k, d>X_{2}} \mu(d) \frac{X}{d} \int_{-\infty}^{\infty} \psi(x) d x \\
& +\sum_{1 \leq|l| \leq L} \sum_{\substack{d \mid k \\
X_{1}<d \leq X_{2}}} \mu(d) \frac{X}{d} \widehat{\psi}\left(\frac{l X}{d}\right)+O_{A}\left(X J^{-A}\right)
\end{aligned}
$$

for any positive constant $A$, and any $L \geq\left(X_{2} / X\right)^{2}$.

The lemma shows that the first term dominates providing that $k \leq X^{1-\varepsilon}$. In general the subsidiary terms will be required.

To prove the lemma we write the sum on the left as

$$
\sum_{d \mid k} \mu(d) \sum_{m=-\infty}^{\infty} \psi\left(\frac{m d}{X}\right)
$$

and we consider separately the cases $d>X_{2}$ and $d \leq X_{2}$. For the former case we have

since $k \geq 2$, and

$$
\sum_{d>X_{2}} \mu(d) \psi(0)=-\psi(0) \sum_{d \leq X_{2}} \mu(d)
$$

$$
\sum_{d>X_{2}} \sum_{m \neq 0}\left|\psi\left(\frac{m d}{X}\right)\right| \ll \sum_{d>X_{2}} \sum_{m \neq 0}\left(\frac{|m| d}{X}\right)^{-A} \ll X\left(X_{2} / X\right)^{1-A} .
$$

For the remaining values of $d$ we apply the Poisson summation formula to obtain

$$
\sum_{d \mid k, d \leq X_{2}} \mu(d) \sum_{m=-\infty}^{\infty} \psi\left(\frac{m d}{X}\right)=\sum_{l=-\infty}^{\infty} \sum_{d \mid k, d \leq X_{2}} \mu(d) \frac{X}{d} \widehat{\psi}\left(\frac{l X}{d}\right) .
$$

Since

and

$$
\sum_{d \mid k, d \leq X_{2}} \frac{\mu(d)}{d}=\frac{\phi(k)}{k}-\sum_{d \mid k, d>X_{2}} \frac{\mu(d)}{d}
$$

$$
\widehat{\psi}(0)=\int_{-\infty}^{\infty} \psi(x) d x
$$

we see that the terms with $l=0$ produce the first and third contributions on the right of (15). When $d \leq X_{1}$ we find that

$$
\sum_{d \leq X_{1}} \sum_{l \neq 0} \frac{X}{d}\left|\widehat{\psi}\left(\frac{l X}{d}\right)\right| \ll \sum_{d \leq X_{1}} \sum_{l \neq 0} \frac{X}{d}\left(\frac{|l| X}{d}\right)^{-A} \ll X\left(X / X_{1}\right)^{-A}
$$


since we have $\widehat{\psi}(x) \ll_{A}|x|^{-A}$ for any positive $A$, by repeated integration by parts. Similarly for $X_{1}<d \leq X_{2}$ we have

$$
\begin{aligned}
\sum_{X_{1}<d \leq X_{2}} \sum_{|l|>L} \frac{X}{d}\left|\widehat{\psi}\left(\frac{l X}{d}\right)\right| & \ll \sum_{X_{1}<d \leq X_{2}} \sum_{|l|>L} \frac{X}{d}\left(\frac{|l| X}{d}\right)^{-A} \\
& \ll X^{1-A} X_{2}^{A} L^{1-A} \ll X\left(X_{2} / X\right)^{2-A} .
\end{aligned}
$$

These estimates combine to yield the formula of the lemma, with a new value for $A$.

The final result of this section is a curiosity concerning Fourier integrals.

LEMMA 14. Let $W(x)$ be an infinitely differentiable function, supported on a compact subset of $(0, \infty)$. Then

$$
\int_{-\infty}^{\infty} \widehat{W}\left(\alpha x^{2}\right) d x=\frac{1-\operatorname{sign}(\alpha) i}{\sqrt{|\alpha|}} \int_{0}^{\infty} W\left(x^{2}\right) d x
$$

for any non-zero real $\alpha$.

For the proof we begin by noting that $\widehat{W}(x) \ll_{A}|x|^{-A}$ for any constant $A$. This will ensure the absolute convergence of all the integrals we shall consider. We shall suppose that $W$ is supported on $[a, b]$, with $0<a<b$. Then the left hand integral is

$$
\begin{aligned}
\lim _{K \rightarrow \infty} \int_{-K}^{K} \widehat{W}\left(\alpha x^{2}\right) d x & =\lim _{K \rightarrow \infty} \int_{-K}^{K} \int_{a}^{b} W(t) e\left(-\alpha x^{2} t\right) d t d x \\
& =\lim _{K \rightarrow \infty} \int_{a}^{b} W(t) \int_{-K}^{K} e\left(-\alpha x^{2} t\right) d x d t
\end{aligned}
$$

by Fubini's Theorem. However,

$$
\int_{K}^{\infty} e\left(-\alpha x^{2} t\right) d x=\left[\frac{e\left(-\alpha x^{2} t\right)}{-4 \pi i \alpha x t}\right]_{K}^{\infty}+\int_{K}^{\infty} e\left(-\alpha x^{2} t\right) \frac{d x}{4 \pi i \alpha x^{2} t} \ll \frac{1}{|\alpha| K t},
$$

by integration by parts. A similar estimate holds for the integral over the range $(-\infty,-K)$. Since $\alpha \neq 0$ is fixed, and $t \geq a>0$ with $a$ fixed, we see that

$$
\int_{a}^{b} W(t) \int_{-K}^{K} e\left(-\alpha x^{2} t\right) d x d t=\int_{a}^{b} W(t) \int_{-\infty}^{\infty} e\left(-\alpha x^{2} t\right) d x d t+O\left(K^{-1}\right)
$$

whence 


$$
\begin{aligned}
\int_{-\infty}^{\infty} \widehat{W}\left(\alpha x^{2}\right) d x & =\int_{a}^{b} W(t) \int_{-\infty}^{\infty} e\left(-\alpha x^{2} t\right) d x d t \\
& =\int_{a}^{b} W(t) \frac{d t}{(1+\operatorname{sign}(\alpha) i) \sqrt{|\alpha| t}}
\end{aligned}
$$

and the result follows on substituting $t=x^{2}$.

4. Proof of Lemma 2. We begin the demonstration of Lemma 2 by expanding

$$
\sum_{m ; s(m)>K}^{\text {odd }} W\left(\frac{m}{M}\right)\left|\sum_{n} a_{n}\left(\frac{m}{n}\right)\right|^{2}
$$

and sorting the resulting terms to produce

$$
\sum_{\Delta} \sum_{s(m)>K}^{\text {odd }} W\left(\frac{m}{M}\right) \sum_{\left(n_{1}, n_{2}\right)=\Delta} a_{n_{1}} \bar{a}_{n_{2}}\left(\frac{m}{n_{1} n_{2}}\right) .
$$

The terms for which $\Delta \leq \Delta_{0}$ are immediately estimated as being at most

$$
\sum_{\Delta \leq \Delta_{0}} \mathcal{C}(M, N, K, \Delta) \sum_{n}\left|a_{n}\right|^{2} .
$$

For any other $\Delta$ the contribution is

$$
\begin{aligned}
& \leq \sum_{s(m)>K}^{\text {odd }} W\left(\frac{m}{M}\right)\left|\sum_{\left(n_{1}, n_{2}\right)=\Delta} a_{n_{1}} \bar{a}_{n_{2}}\left(\frac{m}{n_{1} n_{2}}\right)\right| \\
& \leq \sum_{s(m)>K}^{\text {odd }} W\left(\frac{m}{M}\right)\left|\sum_{\left(n_{1}, n_{2}\right)=1} a_{n_{1} \Delta} \bar{a}_{n_{2} \Delta}\left(\frac{m}{n_{1} n_{2}}\right)\right|
\end{aligned}
$$

on replacing $n_{i}$ by $n_{i} \Delta$. We can pick out the condition $\left(n_{1}, n_{2}\right)=1$ by using a sum involving the Möbius function to give

$$
\begin{aligned}
& \leq \sum_{d} \sum_{s(m)>K}^{\text {odd }} W\left(\frac{m}{M}\right)\left|\sum_{d \mid n_{1}, n_{2}} a_{n_{1} \Delta} \bar{a}_{n_{2} \Delta}\left(\frac{m}{n_{1} n_{2}}\right)\right| \\
& =\sum_{d} \sum_{s(m)>K}^{\text {odd }} W\left(\frac{m}{M}\right)\left|\sum_{d \mid n} a_{n}\left(\frac{m}{n}\right)\right|^{2} \\
& \leq \sum_{d} \mathcal{B}(M, N / \Delta, K) \sum_{d \mid n}\left|a_{n} \Delta\right|^{2} \\
& \leq \mathcal{B}(M, N / \Delta, K) \sum_{n} d(n)\left|a_{n} \Delta\right|^{2} \\
& \leq \mathcal{B}^{*}(M, K) \sum_{n} d(n)\left|a_{n} \Delta\right|^{2},
\end{aligned}
$$


where

$$
\mathcal{B}^{*}(M, K)=\max _{N_{1} \leq N / \Delta_{0}} \mathcal{B}\left(M, N_{1}, K\right) .
$$

Now when we sum over $\Delta>\Delta_{0}$ we get

$$
\begin{aligned}
& \leq \mathcal{B}^{*}(M, K) \sum_{\Delta} \sum_{n} d(n)\left|a_{n \Delta}\right|^{2} \\
& \leq \mathcal{B}^{*}(M, K) \sum_{n} d(n)^{2}\left|a_{n}\right|^{2} \\
& \ll N^{\varepsilon} \mathcal{B}^{*}(M, K) \sum_{n}\left|a_{n}\right|^{2},
\end{aligned}
$$

and the lemma follows.

5. The sum $\Sigma_{3}$. We begin by recalling the definition (12), namely

$$
\Sigma_{3}=\sum_{\left(n_{1}, n_{2}\right)=\Delta} a_{n_{1}} \bar{a}_{n_{2}} \sum_{m}^{\text {odd }} W\left(\frac{m}{M}\right)\left(\frac{m}{n_{1} n_{2}}\right) .
$$

If we set $q=n_{1} n_{2} \Delta^{-2}$ as in Lemma 3 , the inner sum becomes

$$
\sum_{(m, 2 \Delta)=1} W\left(\frac{m}{M}\right)\left(\frac{m}{q}\right)=\sum_{e \mid 2 \Delta} \mu(e)\left(\frac{e}{q}\right) \sum_{m^{\prime}} W\left(\frac{m^{\prime}}{M / e}\right)\left(\frac{m^{\prime}}{q}\right),
$$

and since $q$ will be square-free Lemma 11 shows that this is

$$
\sum_{e \mid 2 \Delta} \mu(e)\left(\frac{e}{q}\right) \frac{M \tau(q)}{e q} \sum_{h=-\infty}^{\infty} \widehat{W}\left(\frac{h}{e q / M}\right)\left(\frac{h}{q}\right) .
$$

When $h=0$ the Jacobi symbol vanishes, since $q \geq N^{2} \Delta^{-2}>1$. The remaining values of $h$ we write in the form $h=a b c^{2}$, where $a= \pm 1$ or \pm 2 , and $b$ is positive, odd and square-free. Thus

$$
\Sigma_{3}=\sum_{e \mid 2 \Delta} \frac{M \mu(e)}{e} \sum_{a} \sum_{c=1}^{\infty} \sum_{\left(n_{1}, n_{2}\right)=1} a_{n_{1}}^{(1)} \bar{a}_{n_{2}}^{(1)} \sum_{b}^{*} \widehat{W}\left(\frac{a b c^{2}}{e n_{1} n_{2} / M}\right)\left(\frac{b}{n_{1} n_{2}}\right),
$$

where

$$
a_{n}^{(1)}=a_{n \Delta} \frac{\tau(n)}{n}\left(\frac{e a c^{2}}{n}\right) .
$$

We first consider the contribution to $\Sigma_{3}$ from terms with $b>K$, namely

$$
\sum_{e \mid 2 \Delta} \frac{M \mu(e)}{e} \sum_{a} \sum_{c} \sum_{\left(n_{1}, n_{2}\right)=1} a_{n_{1}}^{(1)} \bar{a}_{n_{2}}^{(1)} \sum_{b>K}^{*} \widehat{W}\left(\frac{a b c^{2}}{e n_{1} n_{2} / M}\right)\left(\frac{b}{n_{1} n_{2}}\right) .
$$

Since $\widehat{W}(x) \ll_{A}|x|^{-A}$ and $K \geq N^{2} M^{-1}(M N)^{\varepsilon}$, we see that this sum is $O_{\varepsilon}\left(\sum\left|a_{n}\right|^{2}\right)$, providing that we choose $A$ sufficiently large in terms of $\varepsilon$. This is satisfactory for Lemma 3 . 
We now examine the terms in $\Sigma_{3}$ for which $b \leq K$. We break the available range for $b$ into intervals $(B, 2 B]$, with $B=2^{-k} K$. For each such range we have therefore to consider

$$
\sum_{e \mid 2 \Delta} \frac{M \mu(e)}{e} \sum_{a} \sum_{b}^{*} \sum_{\left(n_{1}, n_{2}\right)=1} a_{n_{1}}^{(2)} \bar{a}_{n_{2}}^{(2)} S\left(n_{1} n_{2}\right)\left(\frac{b}{n_{1} n_{2}}\right),
$$

where we now take

$$
a_{n}^{(2)}=a_{n \Delta} \frac{\tau(n)}{n}\left(\frac{e a}{n}\right)
$$

and

$$
S(q)=\sum_{\substack{c=1 \\(c, q)=1}}^{\infty} \widehat{W}\left(\frac{a b c^{2}}{e q / M}\right) .
$$

Since $q>1$ we have $(0, q)=0$, so that

$$
S(q)=\frac{1}{2} \sum_{\substack{c=-\infty \\(c, q)=1}}^{\infty} \widehat{W}\left(\frac{a b c^{2}}{e q / M}\right) .
$$

We may now apply Lemma 13 to $S(q)$, taking $X=\sqrt{e q /(M b)}$ and $\psi(x)=$ $\widehat{W}\left(a x^{2}\right)$. Moreover, we shall take

$$
X_{1}=(M N)^{-\eta / 2} \frac{\sqrt{e}}{\Delta} N(M B)^{-1 / 2} \quad \text { and } \quad X_{2}=(M N)^{\eta} \frac{\sqrt{e}}{\Delta} N(M B)^{-1 / 2} \text {, }
$$

with $\eta>0$, so that we may choose $J=(M N)^{\eta / 4}$ and $L=(M N)^{3 \eta}$. It is then immediately apparent that the error term in (15) makes a negligible contribution to $\Sigma_{3}$, providing that we choose $A$ sufficiently large compared with $\eta$.

The leading term in Lemma 13 will be

$$
\frac{\phi(q)}{q} \sqrt{\frac{e q}{M b}} \int_{-\infty}^{\infty} \widehat{W}\left(a x^{2}\right) d x=\phi(q) \sqrt{\frac{e}{M b q}} \cdot \frac{1-\operatorname{sign}(a) i}{\sqrt{|a|}} \int_{0}^{\infty} W\left(x^{2}\right) d x,
$$

by Lemma 14. To find the corresponding contribution to $\Sigma_{3}$ we must perform the summations over $a$ and $e$, using the observation that

$$
\sum_{e \mid 2 \Delta} \sum_{a= \pm 1, \pm 2} \frac{\mu(e)}{\sqrt{e}}\left(\frac{e a}{q}\right) \frac{1-\operatorname{sign}(a) i}{\sqrt{|a|}}=\frac{\sqrt{q}}{\tau(q)} \kappa(\Delta, q),
$$

where $\kappa(\Delta, q)$ is as given in Lemma 3 . For each range $(B, 2 B]$ we find that the total contribution to $\Sigma_{3}$ is

$$
\frac{1}{2} \sum_{B<b \leq 2 B}^{*} \sqrt{\frac{M}{b}} \sum_{\left(n_{1}, n_{2}\right)=\Delta} a_{n_{1}} \bar{a}_{n_{2}} \frac{\phi(q)}{q} \kappa(\Delta, q)\left(\frac{b}{q}\right) \int_{0}^{\infty} W\left(x^{2}\right) d x,
$$


so that we have

$$
\sum_{b \leq K}^{*} \sqrt{\frac{M}{b}} \sum_{\left(n_{1}, n_{2}\right)=\Delta} a_{n_{1}} \bar{a}_{n_{2}} \frac{\phi(q)}{2 q} \kappa(\Delta, q)\left(\frac{b}{q}\right) \int_{0}^{\infty} W\left(x^{2}\right) d x,
$$

on summing over $B$.

We next examine the error terms arising from the divisor sums in Lemma 13. The first sum contributes

$$
\ll M \Delta^{\eta} \sum_{d \leq X_{2}} \sum_{b}^{*}\left|\sum_{\substack{\left(n_{1}, n_{2}\right)=1 \\ d \mid n_{1} n_{2}}} a_{n_{1}}^{(2)} \bar{a}_{n_{2}}^{(2)}\left(\frac{b}{n_{1} n_{2}}\right)\right|,
$$

for some choice of $e$ and $a$. If we decompose the sum over $d$ into ranges $D<d \leq 2 D$, we can apply Lemma 10 to each, giving a contribution

$$
\ll(M N)^{2 \eta} M N^{-1} \Delta \sqrt{D_{1} D_{2}} \mathcal{B}\left(B, \frac{N}{D_{1} \Delta}\right)^{1 / 2} \mathcal{B}\left(B, \frac{N}{D_{2} \Delta}\right)^{1 / 2} \sum\left|a_{n}\right|^{2} .
$$

There are $O\left(\log X_{2}\right)=O\left((M N)^{\eta}\right)$ ranges $(D, 2 D]$ and $O(\log K)=$ $O\left((M N)^{\eta}\right)$ ranges $(B, 2 B]$, so that the total contribution to $\Sigma_{3}$ is

$$
\ll(M N)^{4 \eta} \frac{M \Delta}{N} \sqrt{D_{1} D_{2}} \mathcal{B}\left(B, \frac{N}{D_{1} \Delta}\right)^{1 / 2} \mathcal{B}\left(B, \frac{N}{D_{2} \Delta}\right)^{1 / 2},
$$

for some choice of $D_{1}, D_{2}$ and $B$. However, if

$$
m_{0}=\min \left\{1, \frac{N / \sqrt{M B}}{D_{1} D_{2}}\right\}
$$

then

$$
1 \ll(M N)^{\eta} m_{0},
$$

since

$$
D_{1} D_{2} \ll X_{2}=(M N)^{\eta} \frac{\sqrt{e}}{\Delta} N(M B)^{-1 / 2} \ll(M N)^{\eta} N(M B)^{-1 / 2} .
$$

Thus (16) is

$$
\ll(M N)^{5 \eta} \frac{M \Delta}{N} m_{0} \sqrt{D_{1} D_{2}} \mathcal{B}\left(B, \frac{N}{D_{1} \Delta}\right)^{1 / 2} \mathcal{B}\left(B, \frac{N}{D_{2} \Delta}\right)^{1 / 2} \sum\left|a_{n}\right|^{2},
$$

which is of the form required for Lemma 3, on setting $\eta=\varepsilon / 5$.

We now consider the contribution from the second divisor sum in (15), for a given range $b \in(B, 2 B]$. In view of the fact that $X=\sqrt{e q /(M b)}$ this will be

$$
\ll M^{1 / 2} B^{-1 / 2} \Delta^{\eta} \sum_{d>X_{2}} d^{-1} \sum_{B<b \leq 2 B}^{*}\left|\sum_{\substack{\left(n_{1}, n_{2}\right)=1 \\ d \mid n_{1} n_{2}}} a_{n_{1}}^{(3)} \bar{a}_{n_{2}}^{(3)}\left(\frac{b}{n_{1} n_{2}}\right)\right| .
$$


Here we have set

$$
a_{n}^{(3)}=a_{n \Delta} \frac{\tau(n)}{\sqrt{n}}\left(\frac{e a}{n}\right)
$$

and we have chosen $e$ and $a$ so as to maximize (17). We again decompose the range for $d$ into subintervals $D<d \leq 2 D$, with $X_{2} \ll D \ll N^{2}$, and apply Lemma 10 for each, to obtain a bound

$$
\ll(M N \Delta)^{\eta} \sqrt{\frac{M}{B D_{1} D_{2}}} \mathcal{B}\left(B, \frac{N}{D_{1} \Delta}\right)^{1 / 2} \mathcal{B}\left(B, \frac{N}{D_{2} \Delta}\right)^{1 / 2} \sum\left|a_{n}\right|^{2} .
$$

As before, there are $O\left((M N)^{\eta}\right)$ possibilities for each of $B$ and $D$, and

$$
\begin{aligned}
\sqrt{\frac{M}{B D_{1} D_{2}}} & \ll \frac{M}{N} \sqrt{D_{1} D_{2}} \min \left\{\Delta, \frac{N / \sqrt{M B}}{D_{1} D_{2}}\right\} \\
& \ll \Delta \frac{M}{N} \sqrt{D_{1} D_{2}} \min \left\{1, \frac{N / \sqrt{M B}}{D_{1} D_{2}}\right\},
\end{aligned}
$$

since

$$
D_{1} D_{2} \gg \frac{X_{2}}{\log ^{2}(2 M N)} \gg N \Delta^{-1}(M B)^{-1 / 2}
$$

It follows that the corresponding contribution to $\Sigma_{3}$ can again be put into the correct form for Lemma 3 by taking $\eta=\varepsilon / 4$.

For the final divisor sum in (15) we have an estimate

$$
\begin{aligned}
& M^{1 / 2} B^{-1 / 2} L \Delta^{\eta} \\
& \quad \times \sum_{X_{1}<d \leq X_{2}} d^{-1} \sum_{b}^{*}\left|\sum_{\substack{\left(n_{1}, n_{2}\right)=1 \\
d \mid n_{1} n_{2}}} a_{n_{1}}^{(3)} \bar{a}_{n_{2}}^{(3)} \widehat{\psi}\left(\frac{l}{d} \sqrt{\frac{e n_{1} n_{2}}{M b}}\right)\left(\frac{b}{n_{1} n_{2}}\right)\right|,
\end{aligned}
$$

for appropriate values of $a, e$ and $l$. We remove the factor $\widehat{\psi}$ by means of Lemma 12 , with $\varrho(x)=\widehat{\psi}(x)$. It follows that $\varrho^{(k)}(x) \ll_{k, A}|x|^{-A}$, as required. Taking $l>0$ for definiteness, our estimate becomes

$$
M^{1 / 2} B^{-1 / 2} L \Delta^{\eta} \sum_{X_{1}<d \leq X_{2}} d^{-1} \sum_{b}^{*}\left|\sum_{\substack{\left(n_{1}, n_{2}\right)=1 \\ d \mid n_{1} n_{2}}} a_{n_{1}}^{(3)} \bar{a}_{n_{2}}^{(3)}\left(\frac{b}{n_{1} n_{2}}\right) I\left(d, b, n_{1}, n_{2}\right)\right| \text {, }
$$

where

$$
I\left(d, b, n_{1}, n_{2}\right)=\frac{1}{2 \pi i} \int_{\sigma-i \infty}^{\sigma+i \infty} \varrho_{+}(s)\left(\frac{l}{d} \sqrt{\frac{e n_{1} n_{2}}{M b}}\right)^{-s} d s .
$$

We therefore obtain a bound

$$
\ll M^{1 / 2+\sigma} B^{-1 / 2+\sigma} X_{2}^{\sigma} L \Delta^{\eta} \int_{-\infty}^{\infty}\left|\varrho_{+}(\sigma+i t)\right| \sum_{X_{1}<d \leq X_{2}} d^{-1} S(\sigma+i t, d) d t,
$$


where

$$
S(s, d)=\sum_{b}^{*}\left|\sum_{\substack{\left(n_{1}, n_{2}\right)=1 \\ d \mid n_{1} n_{2}}} a_{n_{1}}^{(3)} \bar{a}_{n_{2}}^{(3)}\left(\frac{b}{n_{1} n_{2}}\right)\left(n_{1} n_{2}\right)^{-s / 2}\right| .
$$

We may note here that $X_{2} \ll(M N)^{2}$, so that $X_{2}^{\sigma} \ll(M N)^{2 \sigma}$. We again decompose the range for $d$ into subintervals $D<d \leq 2 D$ and apply Lemma 10 for each, observing that

$$
\int_{-\infty}^{\infty}\left|\varrho_{+}(\sigma+i t)\right| d t \ll_{\sigma} 1
$$

Allowing for $O\left((M N)^{\eta}\right)$ values for each of $B$ and $D$, we obtain a total contribution

$$
\begin{aligned}
\ll L(M B)^{\sigma} N^{\eta}(M N)^{3 \eta+2 \sigma} & \\
& \times \sqrt{\frac{M}{B D_{1} D_{2}}} \mathcal{B}\left(B, \frac{N}{D_{1} \Delta}\right)^{1 / 2} \mathcal{B}\left(B, \frac{N}{D_{2} \Delta}\right)^{1 / 2} \sum\left|a_{n}\right|^{2}
\end{aligned}
$$

to $\Sigma_{3}$. This time we have

$$
\sqrt{\frac{M}{B D_{1} D_{2}}} \ll \Delta(M N)^{\eta} \frac{M}{N} \sqrt{D_{1} D_{2}} \min \left\{1, \frac{N / \sqrt{M B}}{D_{1} D_{2}}\right\},
$$

since

$$
D_{1} D_{2} \gg \frac{X_{1}}{\log ^{2}(2 M N)} \gg(M N)^{-\eta} N \Delta^{-1}(M B)^{-1 / 2} .
$$

However, if we choose $\sigma=\eta=\varepsilon / 11$ we have

$$
L(M B)^{\sigma} N^{\eta}(M N)^{4 \eta+2 \sigma} \ll(M N)^{\varepsilon},
$$

in view of the constraint that $B \ll K \ll M$. It follows that our error term can again be put into a form suitable for Lemma 3. This completes the proof of the lemma.

6. The sum $\Sigma_{4}$. We begin by recalling the definition of $\Sigma_{4}$, given by (13) as

$$
\Sigma_{4}=\sum_{\left(n_{1}, n_{2}\right)=\Delta} a_{n_{1}} \bar{a}_{n_{2}} \sum_{m ; s(m) \leq K}^{*} W\left(\frac{m}{M}\right)\left(\frac{m}{n_{1} n_{2}}\right) .
$$

We put $m=u^{2} v$ with $v$ square-free, and we write $q=n_{1} n_{2} \Delta^{-2}$ as usual. Then

$$
\Sigma_{4}=\sum_{\left(n_{1}, n_{2}\right)=\Delta} a_{n_{1}} \bar{a}_{n_{2}} \sum_{\substack{1 \leq v \leq K \\(v, 2 \Delta)=1}}^{*}\left(\frac{v}{q}\right) \Sigma_{5}(v, q),
$$


where

$$
\Sigma_{5}(v, q)=\sum_{\substack{u=1 \\(u, 2 q \Delta)=1}}^{\infty} \psi\left(\frac{u}{\sqrt{M / v}}\right)=\frac{1}{2} \sum_{\substack{u=-\infty \\(u, 2 q \Delta)=1}}^{\infty} \psi\left(\frac{u}{\sqrt{M / v}}\right),
$$

with $\psi(x)=W\left(x^{2}\right)$. The final sum is of the form needed for an application of Lemma 13. We break the available range for $v$ into subintervals $(B, 2 B]$. For each such range we apply Lemma 13 with $X=\sqrt{M / v}$ and

$$
X_{1}=(M N)^{-\eta} M^{1 / 2} B^{-1 / 2} \quad \text { and } \quad X_{2}=(M N)^{\eta} M^{1 / 2} B^{-1 / 2},
$$

with $\eta>0$, so that we may choose $J=(M N)^{\eta / 2}$ and $L=(M N)^{3 \eta}$. It is then immediately apparent that the error term in (15) makes a negligible contribution to $\Sigma_{4}$, providing that we choose $A$ sufficiently large compared with $\eta$.

We begin by examining the leading terms. For each range $(B, 2 B]$ these are

$$
\frac{1}{2} \sum_{\substack{B<v \leq 2 B \\(v, 2 \Delta)=1}}^{*} \sqrt{\frac{M}{v}} \sum_{\left(n_{1}, n_{2}\right)=\Delta} a_{n_{1}} \bar{a}_{n_{2}} \frac{\phi(2 q \Delta)}{2 q \Delta}\left(\frac{v}{q}\right) \int_{-\infty}^{\infty} \psi(x) d x .
$$

Since

$$
\frac{1}{2} \int_{-\infty}^{\infty} \psi(x) d x=\int_{0}^{\infty} W\left(x^{2}\right) d x
$$

the leading terms are of the correct form for Lemma 4, after combining the intervals $(B, 2 B]$ to produce $[1, K]$.

We now consider the sums over divisors in Lemma 13. Since $\psi(0)=$ $W(0)=0$ the first sum vanishes. The second sum contributes to $\Sigma_{4}$ a total

$$
\ll \sqrt{\frac{M}{B}} \sum_{d>X_{2}} d^{-1} \sum_{B<v \leq 2 B}^{*}\left|\sum_{\substack{\left(n_{1}, n_{2}\right)=\Delta \\ d \mid 2 q \Delta}} a_{n_{1}} \bar{a}_{n_{2}}\left(\frac{v}{q}\right)\right| .
$$

We replace $n_{i}$ by $n_{i} \Delta$ and we write $d=(d, 2 \Delta) e$. Then each value of $e$ can arise from $O\left(N^{\eta}\right)$ values of $d$, so that the above bound becomes

$$
\ll N^{\eta} \sqrt{\frac{M}{B}} \sum_{e>X_{2} / 2 \Delta} e^{-1} \sum_{B<v \leq 2 B}^{*}\left|\sum_{\substack{\left(n_{1}, n_{2}\right)=1 \\ e \mid n_{1} n_{2}}} a_{n_{1} \Delta} \bar{a}_{n_{2} \Delta}\left(\frac{v}{n_{1} n_{2}}\right)\right| .
$$

We break the range for $e$ into intervals $D<e \leq 2 D$, where $X_{2} / \Delta \ll D \ll$ $N^{2}$. We can then apply Lemma 10 to give a bound

$$
\ll(M N)^{3 \eta} \sqrt{\frac{M}{B D_{1} D_{2}}} \mathcal{B}\left(B, \frac{N}{D_{1} \Delta}\right)^{1 / 2} \mathcal{B}\left(B, \frac{N}{D_{2} \Delta}\right)^{1 / 2} \sum\left|a_{n}\right|^{2},
$$


where

$$
D_{1} D_{2} \gg \frac{X_{2}}{\Delta \log ^{2}(2 M N)} .
$$

There remains the third divisor sum in Lemma 13. When $v$ is in a given range $B<v \leq 2 B$ the corresponding contribution to $\Sigma_{4}$ is

$$
\frac{1}{2} \sum_{B<v \leq 2 B}^{*} \sum_{1 \leq|l| \leq L} \sum_{X_{1}<d \leq X_{2}} \mu(d) \frac{\sqrt{M / v}}{d} \widehat{\psi}\left(\frac{l \sqrt{M / v}}{d}\right) S(d, v),
$$

where

$$
S(d, v)=\sum_{\left(n_{1}, n_{2}\right)=\Delta, d \mid 2 q \Delta} a_{n_{1}} \bar{a}_{n_{2}}\left(\frac{v}{q}\right) .
$$

Since $\widehat{\psi}(x) \ll 1$ we obtain a bound

$$
\ll L \sum_{X_{1}<d \leq X_{2}} \frac{\sqrt{M / B}}{d} \sum_{B<v \leq 2 B}^{*}|S(d, v)| .
$$

This may now be handled as before, to give an estimate

$$
\ll(M N)^{5 \eta} \sqrt{\frac{M}{B D_{1} D_{2}}} \mathcal{B}\left(B, \frac{N}{D_{1} \Delta}\right)^{1 / 2} \mathcal{B}\left(B, \frac{N}{D_{2} \Delta}\right)^{1 / 2} \sum\left|a_{n}\right|^{2},
$$

where

$$
D_{1} D_{2} \gg \frac{X_{1}}{\Delta \log ^{2}(2 M N)}
$$

We then see that both this bound and (18) are acceptable for Lemma 4, after choosing $\eta=\varepsilon / 5$.

7. Comparison of the leading terms. This section is devoted to the proof of Lemma 5. We begin with the observation that

$$
\kappa(\Delta, q)=\sum_{e \mid \Delta} \frac{\mu(e)}{\sqrt{e}}\left(\frac{e}{q}\right)
$$

so that

$$
\sum_{b \leq K}^{*} \frac{1}{\sqrt{b}} \kappa(\Delta, q)\left(\frac{b}{q}\right)=\sum_{w} \frac{\alpha(w)}{\sqrt{w}}\left(\frac{w}{q}\right)
$$

where

$$
\alpha(w)=\sum_{w=b e} \mu(e)
$$

subject to the conditions

$$
e \mid \Delta, \quad b \leq K, \quad b \text { odd and square-free. }
$$


Similarly we have

$$
\frac{\phi(q \Delta)}{2 q \Delta}=\frac{\phi(q)}{2 q} \cdot \frac{\phi(\Delta)}{\Delta}
$$

and

$$
\frac{\phi(\Delta)}{\Delta}=\sum_{u \mid \Delta} \frac{\mu(u)}{\sqrt{u^{2}}}\left(\frac{u^{2}}{q}\right)
$$

since $(q, \Delta)=1$. Thus

$$
\sum_{\substack{v \leq K \\(v, 2 \Delta)=1}}^{*} \frac{1}{\sqrt{v}} \cdot \frac{\phi(\Delta)}{\Delta}\left(\frac{v}{q}\right)=\sum_{w} \frac{\beta(w)}{\sqrt{w}}\left(\frac{w}{q}\right),
$$

where

$$
\beta(w)=\sum_{w=v u^{2}} \mu(u)
$$

subject to the conditions

$$
u \mid \Delta, \quad v \leq K, \quad(v, 2 \Delta)=1, \quad v \text { square-free. }
$$

We see at once that if $\alpha(w) \neq 0$ then $w$ is odd, $w \leq K \Delta$ and $w=r s^{2}$ with $r$ square-free and $s \mid \Delta$. The same is true for $\beta(w)$ except that the upper bound is now $w \leq K \Delta^{2}$. Moreover, if $w$ is odd and $w \leq K$, then

$$
\alpha(w)=\sum_{e \mid(w, \Delta)} \mu(e) \mu^{2}(w / e)
$$

and

$$
\beta(w)=\sum_{\substack{w=v u^{2}, u \mid \Delta \\(v, \Delta)=1}} \mu(u) \mu^{2}(v) .
$$

The two expressions on the right are each multiplicative functions of $w$, and it is an elementary exercise to show that they are identically equal. It follows that the function $\gamma(w)=\alpha(w)-\beta(w)$ is supported on the interval

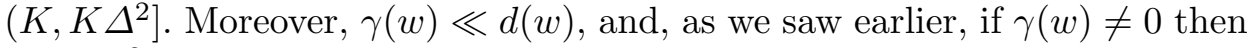
$w=r s^{2}$ with $r$ square-free and $s \mid \Delta$. It should also be observed that the function $\gamma$ is independent of $q$. We now see that

$$
\begin{aligned}
M_{3}-M_{4} \ll & M^{1 / 2} \sum_{w} \frac{|\gamma(w)|}{\sqrt{w}}\left|\sum_{\left(n_{1}, n_{2}\right)=\Delta} a_{n_{1}} \bar{a}_{n_{2}} \frac{\phi(q)}{q}\left(\frac{w}{q}\right)\right| \\
\ll & M^{1 / 2} K^{-1 / 2}(M N)^{\eta} \\
& \quad \times \sum_{s \mid \Delta} \sum_{K \Delta^{-2}<r \leq K \Delta^{2}}^{*} \mid \sum_{\left(n_{1}, n_{2}\right)=\Delta} a_{n_{1}} \bar{a}_{n_{2}} \frac{\phi(q)}{q}\left(\frac{r s^{2}}{q}\right) .
\end{aligned}
$$


We write, temporarily, $n_{i}=m_{i} \Delta$ and we set

$$
a_{n}^{(4)}=\frac{\phi(n)}{n} a_{n \Delta}
$$

if $(n, s)=1$ and $a_{n}^{(4)}=0$ otherwise. We then decompose the range for $r$ into subintervals $R<r \leq 2 R$, so that

$$
M_{3}-M_{4} \ll M^{1 / 2} K^{-1 / 2}(M N)^{2 \eta} \sum_{R<r \leq 2 R}^{*}\left|\sum_{\left(m_{1}, m_{2}\right)=1} a_{m_{1}}^{(4)} \bar{a}_{m_{2}}^{(4)}\left(\frac{r}{m_{1} m_{2}}\right)\right|,
$$

for appropriate values of $s$ and $R$. We may now apply Lemma 10 to show that

$$
M_{3}-M_{4} \ll M^{1 / 2} K^{-1 / 2}(M N)^{3 \eta} \mathcal{B}\left(R, N^{\prime}\right) \sum\left|a_{n}\right|^{2},
$$

for some $N^{\prime} \ll N(M N)^{\eta}$. Finally, an application of Lemma 9 allows us to replace $R$ by $K \Delta^{2}(M N)^{4 \eta}$, and $N^{\prime}$ by $N(M N)^{4 \eta}$ with the loss of a further factor $(M N)^{\eta}$. Lemma 5 now follows, on setting $\eta=\varepsilon / 4$.

8. The recursive estimates. In this section we shall prove the recursive estimates given by Lemmas 6,7 and 8 . We remark at the outset that although Lemma 6 assumes that $N \leq M$, we will still have

$$
\mathcal{B}(M, N) \ll_{\varepsilon}(M N)^{\varepsilon}\left(M+N^{\xi}\right)
$$

when $M \leq N$. To see this we apply Lemma 1 , which shows that

$$
\mathcal{B}(M, N) \ll \mathcal{B}(N, M) \ll_{\varepsilon}(M N)^{\varepsilon}\left(N+M^{\xi}\right) \ll_{\varepsilon}(M N)^{\varepsilon}\left(M+N^{\xi}\right),
$$

on noting that $N$ and $M^{\xi}$ are both at most $N^{\xi}$. Similarly we can show that

$$
\mathcal{B}(M, N) \ll_{\varepsilon}(M N)^{\varepsilon}\left(N+M^{\xi}\right) .
$$

We may also observe that the conditions $M, N \geq 1$ are redundant, since the bound given is trivial when $M$ or $N$ is less than 1 . (Indeed, $\mathcal{B}(M, N)=0$ when either $M$ or $N$ is less than $1 / 2$.)

For the proof of Lemma 6 we combine Lemmas 3, 4 and 5, using our assumption about $\mathcal{B}(M, N)$ to bound the error terms that arise. We begin by investigating the error term $E_{3}$, so that we must consider

$$
\begin{aligned}
\Delta(M N)^{\varepsilon} & \frac{M}{N} \sqrt{D_{1} D_{2}} \\
& \times \min \left\{1, \frac{N / \sqrt{M B}}{D_{1} D_{2}}\right\} \mathcal{B}\left(B, \frac{N}{D_{1} \Delta}\right)^{1 / 2} \mathcal{B}\left(B, \frac{N}{D_{2} \Delta}\right)^{1 / 2},
\end{aligned}
$$

where $1 \ll B \ll K$ and $D_{1}, D_{2} \gg(M N)^{-\varepsilon}$. Then, according to our hypothesis, we have 


$$
\begin{aligned}
\mathcal{B}\left(B, \frac{N}{D_{1} \Delta}\right)^{1 / 2} \mathcal{B} & \left(B, \frac{N}{D_{2} \Delta}\right)^{1 / 2} \\
& \ll(B N)^{2 \varepsilon}\left(B^{\xi}+N / D_{1}\right)^{1 / 2}\left(B^{\xi}+N / D_{2}\right)^{1 / 2} \\
& \ll(M N)^{\varepsilon}\left(B^{\xi}+B^{\xi / 2} N^{1 / 2}+N D_{1}^{-1 / 2} D_{2}^{-1 / 2}\right) .
\end{aligned}
$$

Since

we find that

$$
\min \left\{1, \frac{N / \sqrt{M B}}{D_{1} D_{2}}\right\} \leq \frac{N / \sqrt{M B}}{D_{1} D_{2}}
$$

$$
\frac{M}{N} \sqrt{D_{1} D_{2}} \min \left\{1, \frac{N / \sqrt{M B}}{D_{1} D_{2}}\right\} B^{\xi} \ll(M N)^{\varepsilon} M^{1 / 2} B^{\xi-1 / 2} .
$$

Similarly, since

$$
\min \left\{1, \frac{N / \sqrt{M B}}{D_{1} D_{2}}\right\} \leq\left(\frac{N / \sqrt{M B}}{D_{1} D_{2}}\right)^{1 / 2}
$$

we have

$$
\frac{M}{N} \sqrt{D_{1} D_{2}} \min \left\{1, \frac{N / \sqrt{M B}}{D_{1} D_{2}}\right\} B^{\xi / 2} N^{1 / 2} \leq M^{3 / 4} B^{\xi / 2-1 / 4} .
$$

Finally, since

$$
\min \left\{1, \frac{N / \sqrt{M B}}{D_{1} D_{2}}\right\} \leq 1
$$

we have

$$
\frac{M}{N} \sqrt{D_{1} D_{2}} \min \left\{1, \frac{N / \sqrt{M B}}{D_{1} D_{2}}\right\} N\left(D_{1} D_{2}\right)^{-1 / 2} \leq M .
$$

However,

$$
M^{3 / 4} B^{\xi / 2-1 / 4}=\left\{M^{1 / 2} B^{\xi-1 / 2}\right\}^{1 / 2} M^{1 / 2} \ll M^{1 / 2} B^{\xi-1 / 2}+M,
$$

whence (19) may be estimated as

$$
\ll \Delta(M N)^{3 \varepsilon}\left(M^{1 / 2} B^{\xi-1 / 2}+M\right) .
$$

After replacing $\varepsilon$ by $\varepsilon / 3$ this will be satisfactory for Lemma 6 , in view of the constraint that $B \ll K$.

We now turn to the error $E_{4}$ occurring in Lemma 4 . Here we investigate

$$
(M N)^{\varepsilon}\left(\frac{M}{B D_{1} D_{2}}\right)^{1 / 2} \mathcal{B}\left(B, \frac{N}{D_{1} \Delta}\right)^{1 / 2} \mathcal{B}\left(B, \frac{N}{D_{2} \Delta}\right)^{1 / 2},
$$

with parameters $B, D_{1}, D_{2} \gg(M N)^{-\varepsilon}$, subject to $B \ll K$ and

$$
D_{1} D_{2} \gg(M N)^{-\varepsilon} \Delta^{-1} M^{1 / 2} B^{-1 / 2} \text {. }
$$


We shall use (20) as before. Since $D_{1} D_{2} \gg(M N)^{-2 \varepsilon}$ the first term contributes

$$
\ll(M N)^{3 \varepsilon} M^{1 / 2} B^{\xi-1 / 2} .
$$

For the remaining terms we use the lower bound for $D_{1} D_{2}$ given by (22), leading to a contribution

$$
\ll(M N)^{3 \varepsilon}\left(\Delta^{1 / 2} M^{1 / 4} N^{1 / 2} B^{\xi / 2-1 / 4}+\Delta N\right) .
$$

However,

$$
M^{1 / 4} N^{1 / 2} B^{\xi / 2-1 / 4}=\left\{M^{1 / 2} B^{\xi-1 / 2}\right\}^{1 / 2} N^{1 / 2} \ll M^{1 / 2} B^{\xi-1 / 2}+N,
$$

whence (21) can be estimated as

$$
\ll \Delta(M N)^{3 \varepsilon}\left(M^{1 / 2} B^{\xi-1 / 2}+N\right) .
$$

This too is satisfactory for Lemma 6 , in view of the constraint that $B \ll K$.

Finally, we consider the contribution from Lemma 5, which is

$$
\begin{aligned}
M^{1 / 2} K^{-1 / 2}(M N)^{\varepsilon} \mathcal{B} & \left.K \Delta^{2}(M N)^{\varepsilon}, N(M N)^{\varepsilon}\right) \\
& \ll(M N)^{4 \varepsilon} M^{1 / 2} K^{-1 / 2}\left\{\left(K \Delta^{2}\right)^{\xi}+N\right\} \\
& \ll \Delta^{4}(M N)^{4 \varepsilon}\left(M^{1 / 2} K^{\xi-1 / 2}+M^{1 / 2} N K^{-1 / 2}\right),
\end{aligned}
$$

since $\xi \leq 2$. This is again satisfactory for Lemma 6 .

We now turn to the proof of Lemma 7. When we feed Lemma 6 into Lemma 2 we find that

$$
\begin{aligned}
& \mathcal{B}(M, N, K) \\
& \quad \ll(M N)^{\varepsilon}\left\{\mathcal{B}\left(M, N_{1}, K\right)+\Delta_{0}^{5}\left(M+N+M^{1 / 2} K^{\xi-1 / 2}+M^{1 / 2} N K^{-1 / 2}\right)\right\},
\end{aligned}
$$

with $N_{1} \leq N / \Delta_{0}$, providing that $1 \leq \Delta_{0}<N$ and

$$
N^{2} M^{-1}(M N)^{\varepsilon} \leq K \leq M(M N)^{-\varepsilon} .
$$

It follows that if $1 \leq \Delta_{0}<N \leq N_{0}$ and

$$
N_{0}^{2} M^{-1}\left(M N_{0}\right)^{\varepsilon} \leq K \leq M\left(M N_{0}\right)^{-\varepsilon},
$$

then

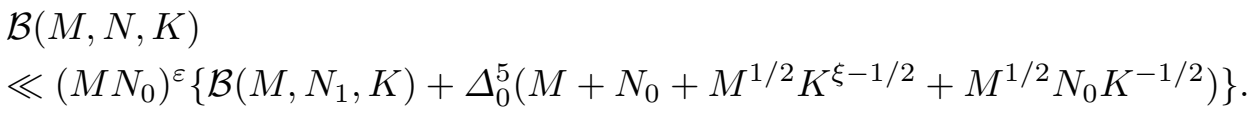

We shall choose $K=N_{0}^{2} M^{-1}\left(M N_{0}\right)^{\varepsilon}$, which is admissible providing that

$$
N_{0}\left(M N_{0}\right)^{\varepsilon} \leq M \text {. }
$$

Our bound now becomes

$$
\mathcal{B}(M, N, K) \ll\left(M N_{0}\right)^{3 \varepsilon}\left\{\mathcal{B}\left(M, N_{1}, K\right)+\Delta_{0}^{5}\left(M+M^{1-\xi} N_{0}^{2 \xi-1}\right)\right\},
$$


since $\xi \leq 2$. We now choose a large integer $R$, and we set $\Delta_{0}=N_{0}^{1 / R}$. With this choice we apply (24) to derive successive estimates

$$
\mathcal{B}\left(M, N_{r-1}, K\right) \ll\left(M N_{0}\right)^{3 \varepsilon}\left\{\mathcal{B}\left(M, N_{r}, K\right)+\Delta_{0}^{5}\left(M+M^{1-\xi} N_{0}^{2 \xi-1}\right)\right\},
$$

with $N_{r} \leq \Delta_{0}^{-r} N_{0}$. We terminate the process at $r=r_{0}$, where

$$
N_{r_{0}} \leq \Delta_{0}<N_{r_{0}-1} .
$$

Our choice of $\Delta_{0}$ ensures that this occurs for $r_{0} \leq R$. On combining our estimates we find that

$$
\mathcal{B}\left(M, N_{0}, K\right) \ll_{R}\left(M N_{0}\right)^{3 R \varepsilon}\left\{\mathcal{B}\left(M, N_{r_{0}}, K\right)+\Delta_{0}^{5}\left(M+M^{1-\xi} N_{0}^{2 \xi-1}\right)\right\} .
$$

The bound (25) yields

$$
\mathcal{B}\left(M, N_{r_{0}}, K\right) \ll M \Delta_{0}
$$

in view of the trivial estimate $\mathcal{B}(M, N, K) \ll M N$. We therefore deduce that

$$
\begin{aligned}
\mathcal{B}\left(M, N_{0}, K\right) & \ll_{R}\left(M N_{0}\right)^{3 R \varepsilon} \Delta_{0}^{5}\left(M+M^{1-\xi} N_{0}^{2 \xi-1}\right) \\
& \ll_{R}\left(M N_{0}\right)^{3 R \varepsilon} N_{0}^{5 / R}\left(M+M^{1-\xi} N_{0}^{2 \xi-1}\right),
\end{aligned}
$$

subject to (23). Since $\mathcal{B}\left(M, N_{0}\right) \leq \mathcal{B}\left(M, N_{0}, K\right)$, we also have the corresponding bound for $\mathcal{B}\left(M, N_{0}\right)$. When (23) fails we can simply use the bound

$$
\begin{aligned}
\mathcal{B}\left(M, N_{0}\right) & \ll\left(M N_{0}\right)^{\varepsilon}\left(M+N_{0}^{\xi}\right) \\
& =\left(M N_{0}\right)^{\varepsilon}\left(M+M^{1-\xi} N_{0}^{2 \xi-1}\left(M / N_{0}\right)^{\xi-1}\right) \\
& \ll\left(M N_{0}\right)^{2 \varepsilon}\left(M+M^{1-\xi} N_{0}^{2 \xi-1}\right) .
\end{aligned}
$$

Since $R$ can be chosen arbitrarily large Lemma 7 follows.

It remains to deduce Lemma 8 from Lemma 7 . The result is immediate when $N^{(2 \xi-1) / \xi} \leq M$, since $M^{1-\xi} N^{2 \xi-1} \leq N^{(2 \xi-1) / \xi}$ in this case. In the alternative case Lemmas 7 and 9 yield

$$
\begin{aligned}
\mathcal{B}(M, N) & \ll \mathcal{B}\left(N^{(2 \xi-1) / \xi}(M N)^{\varepsilon}, N\right) \\
& \ll(M N)^{2 \varepsilon}\left\{N^{(2 \xi-1) / \xi}+\left(N^{(2 \xi-1) / \xi}\right)^{1-\xi} N^{2 \xi-1}\right\} \\
& \ll(M N)^{2 \varepsilon} N^{(2 \xi-1) / \xi}
\end{aligned}
$$

and the result again follows.

9. Proof of the corollaries. For the proof of Corollary 1 we begin by observing that

$$
\sum_{n \leq N} \mu^{2}(n) a_{n} \chi(n)=\sum_{n \leq N}^{*} a_{n} \chi(n)+\chi(2) \sum_{n \leq N / 2}^{*} a_{2 n} \chi(n),
$$


so that Cauchy's inequality yields

$$
\left|\sum_{n \leq N} \mu^{2}(n) a_{n} \chi(n)\right|^{2} \ll\left|\sum_{n \leq N}^{*} a_{n} \chi(n)\right|^{2}+\left|\sum_{n \leq N / 2}^{*} a_{2 n} \chi(n)\right|^{2} .
$$

It follows that it will be sufficient to prove Corollary 1 when $a_{n}$ is supported on the odd square-free integers. We now break the sum over $\chi \in S(Q)$ into four parts corresponding to characters $\chi$ of the form $\left(\frac{n}{m}\right)$ or $\left(\frac{n}{m}\right)\left(\frac{-1}{n}\right)$ or $\left(\frac{n}{m}\right)\left(\frac{2}{n}\right)$ or $\left(\frac{n}{m}\right)\left(\frac{-2}{n}\right)$. We replace $a_{n}$ by $a_{n}^{\prime}=a_{n}$ or $a_{n}\left(\frac{-1}{n}\right)$ or $a_{n}\left(\frac{2}{n}\right)$ or $a_{n}\left(\frac{-2}{n}\right)$ accordingly, and apply Theorem 1 to each part. The result then follows.

To derive Corollary 2 from Corollary 1 we write $f=f_{\chi}$ for the conductor of $\chi$. Thus if $n=k r^{2}$ with $k$ square-free, we have

$$
a_{n} \chi(n)=a_{k r^{2}} \chi(k)\left(\sum_{h \mid(f, r)} \mu(h)\right) .
$$

It therefore follows by Cauchy's inequality that

$$
\left|\sum_{n \leq N} a_{n} \chi(n)\right|^{2} \leq d(f) \sum_{h \mid f}\left|\sum_{r: h \mid r} \sum_{k \leq N r^{-2}} \mu^{2}(k) a_{k r^{2}} \chi(k)\right|^{2} .
$$

We now write

$$
b_{k, h}=\sum_{r: h \mid r} a_{k r^{2}}
$$

so that

$$
\left|\sum_{n \leq N} a_{n} \chi(n)\right|^{2} \ll Q^{\varepsilon} \sum_{h=1}^{\infty}\left|\sum_{k} \mu^{2}(k) b_{k, h} \chi(k)\right|^{2} .
$$

Since the sequence $a_{n}$ is supported on the range $[1, N]$ the summation over $k$ can be taken to run over $1 \leq k \leq N$. We can now use Corollary 1 for each value of $h$, to obtain

$$
\sum_{\chi \in S(Q)}\left|\sum_{n \leq N} a_{n} \chi(n)\right|^{2} \ll_{\varepsilon}(Q N)^{\varepsilon}(Q+N) \sum_{h} \sum_{k \leq N} \mu^{2}(k)\left|b_{k, h}\right|^{2} .
$$

However,

$$
\sum_{k \leq N} \mu^{2}(k)\left|b_{k, h}\right|^{2}=\sum_{n_{1} n_{2}=\square, h^{2} \mid\left(n_{1}, n_{2}\right)}\left|a_{n_{1}} a_{n_{2}}\right|,
$$

whence

$$
\sum_{h} \sum_{k \leq N} \mu^{2}(k)\left|b_{k, h}\right|^{2} \leq \sum_{n_{1} n_{2}=\square}\left|a_{n_{1}} a_{n_{2}}\right| \sum_{h \mid n_{1}} 1 \ll N^{\varepsilon} \sum_{n_{1} n_{2}=\square}\left|a_{n_{1}} a_{n_{2}}\right| .
$$

The required result then follows. 
It is now an easy matter to derive Corollary 3 . It suffices to show that

$$
\sum_{n_{1} n_{2}=\square} 1 \ll N^{1+\varepsilon}
$$

However, if $n_{1} n_{2}$ is a square, then we must have $n_{1}=k r^{2}$ and $n_{2}=k s^{2}$ for some $k, r, s$. Hence

$$
\sum_{n_{1} n_{2}=\square} 1 \leq \sum_{k \leq N}\left(\sqrt{\frac{N}{k}}\right)^{2} \ll N \log N
$$

and the result follows.

Finally, we consider Corollary 4. We first examine the case in which $m$ is restricted to be square-free. Since $\left|a_{m}\right|,\left|b_{n}\right| \leq 1$ we have

$$
\begin{aligned}
\sum_{m \leq M}^{*} \sum_{n \leq N} a_{m} b_{n}\left(\frac{n}{m}\right) & \ll \sum_{m \leq M}^{*}\left|\sum_{n \leq N} b_{n}\left(\frac{n}{m}\right)\right| \\
& \ll M^{1 / 2}\left(\sum_{m \leq M}^{*}\left|\sum_{n \leq N} b_{n}\left(\frac{n}{m}\right)\right|^{2}\right)^{1 / 2} \\
& \ll M^{1 / 2}\left(M^{\varepsilon} N^{1+\varepsilon}(M+N)\right)^{1 / 2},
\end{aligned}
$$

by Cauchy's inequality and Corollary 3 . This establishes Corollary 4 when $m$ is restricted to be square-free. In general we have

$$
\begin{aligned}
\sum_{m \leq M}^{\text {odd }} \sum_{n \leq N} a_{m} b_{n}\left(\frac{n}{m}\right) & =\sum_{r \leq M^{1 / 2}} \sum_{k \leq M r^{-2}}^{*} \sum_{n \leq N} a_{k r^{2}} b_{n, r}\left(\frac{n}{k}\right) \\
& \ll \sum_{r \leq M^{1 / 2}}(M N)^{\varepsilon}\left(\left(\frac{M}{r^{2}}\right) N^{1 / 2}+\left(\frac{M}{r^{2}}\right)^{1 / 2} N\right) \\
& \ll(M N)^{\varepsilon}\left(M N^{1 / 2}+M^{1 / 2} N\right) \log M,
\end{aligned}
$$

where $b_{n, r}=b_{n}$ if $(n, r)=1$ and $b_{n, r}=0$ otherwise. The required result then follows.

10. Proof of Theorem 2. For the proof of Theorem 2 we shall write

$$
S(Q, s)=\sum_{\chi \in S(2 Q)-S(Q)}|L(s, \chi)|^{4}
$$

and we define $\nu(\sigma)$ to be the infimum of those exponents $\nu$ for which

$$
S(Q, \sigma+i t) \ll\left(Q+(Q T)^{2-2 \sigma}\right)(Q T)^{\nu},
$$

uniformly in $Q$ and $t$. Here we have written $T=|t|+1$ for convenience of notation. 
We begin by using the formula

$$
\begin{aligned}
L(s, \chi)^{2}= & \sum_{n=1}^{\infty} d(n) \chi(n) n^{-s} e^{-n / U} \\
& -\frac{1}{2 \pi i} \int_{\alpha-i \infty}^{\alpha+i \infty} L(w, \chi)^{2} \Gamma(w-s) U^{w-s} d w,
\end{aligned}
$$

which will be valid for $0 \leq \alpha<\sigma=\Re(s) \leq 1$. In view of the bound

$$
\Gamma(x+i y) \ll_{x} e^{-|y|},
$$

Cauchy's inequality leads to the estimate

$$
\begin{aligned}
S(Q, s) \ll & \sum_{\chi \in S(2 Q)-S(Q)}\left|\sum_{n=1}^{\infty} d(n) \chi(n) n^{-s} e^{-n / U}\right|^{2} \\
& +U^{2(\alpha-\sigma)} \int_{-\infty}^{\infty} S(Q, \alpha+i \tau) e^{-|\tau-t|} d \tau,
\end{aligned}
$$

for fixed $\sigma$ and $\alpha$, where $s=\sigma+i t$. The functional equation shows that

$$
|L(\alpha+i \tau, \chi)|^{4} \ll(Q T)^{2-4 \alpha}|L(1-\alpha+i \tau, \chi)|^{4},
$$

whence

$$
S(Q, \alpha+i \tau) \ll\left(Q T_{\tau}\right)^{2-4 \alpha}\left(Q+\left(Q T_{\tau}\right)^{2 \alpha}\right)\left(Q T_{\tau}\right)^{\nu(1-\alpha)+\varepsilon},
$$

where $T_{\tau}=|\tau|+1$. It therefore follows that

$$
\begin{aligned}
S(Q, s) \ll & \sum_{\chi \in S(2 Q)-S(Q)}\left|\sum_{n=1}^{\infty} d(n) \chi(n) n^{-s} e^{-n / U}\right|^{2} \\
& +U^{2(\alpha-\sigma)}(Q T)^{2-4 \alpha}\left\{Q+(Q T)^{2 \alpha}\right\}(Q T)^{\nu(1-\alpha)+\varepsilon},
\end{aligned}
$$

for any $\varepsilon>0$. To handle the sum on the right we first observe that the contribution from terms with $n>N_{0}=U \log ^{2} Q T$ will be negligible. We break the remaining sum into $O\left(\log N_{0}\right)$ ranges $N<n \leq 2 N$ with $N \ll N_{0}$, and apply Cauchy's inequality. Since

$$
\sum_{\chi \in S(2 Q)-S(Q)}\left|\sum_{N<n \leq 2 N} d(n) \chi(n) n^{-s} e^{-n / U}\right|^{2} \ll Q^{\varepsilon} N^{1-2 \sigma+\varepsilon}(Q+N),
$$

by Corollary 3, we deduce that

$$
\sum_{\chi \in S(2 Q)-S(Q)}\left|\sum_{n=1}^{\infty} d(n) \chi(n) n^{-s} e^{-n / U}\right|^{2} \ll(Q T U)^{\varepsilon}\left(Q+U^{2-2 \sigma}\right),
$$

for $1 / 2 \leq \sigma \leq 1$. On comparing estimates we see that 


$$
\begin{aligned}
S(Q, s) \ll & (Q T U)^{\varepsilon}\left\{Q+U^{2-2 \sigma}\right\} \\
& +U^{2(\alpha-\sigma)}(Q T)^{2-4 \alpha}\left\{Q+(Q T)^{2 \alpha}\right\}(Q T)^{\nu(1-\alpha)+\varepsilon},
\end{aligned}
$$

for $1 / 2 \leq \sigma \leq 1$ and $0 \leq \alpha<\sigma$.

To prove Theorem 2 we first consider the case in which $\sigma>1 / 2$. We pick $\alpha=1-\sigma$, so that (27) yields

$S(Q, s) \ll(Q T U)^{\varepsilon}\left\{\left(Q+U^{2-2 \sigma}\right)+\left(Q T U^{-1}\right)^{4 \sigma-2}\left(Q+(Q T)^{2-2 \sigma}\right)(Q T)^{\nu(\sigma)}\right\}$.

On taking $U=(Q T)^{1+\delta}$, with $0<\delta<1$, we therefore see that

$$
S(Q, s) \ll(Q T)^{3 \varepsilon}\left\{Q+(Q T)^{2-2 \sigma}\right\}\left\{(Q T)^{\delta}+(Q T)^{\nu(\sigma)-(4 \sigma-2) \delta}\right\}
$$

uniformly in $t$. Since $\varepsilon$ is arbitrary it follows from the definition of $\nu(\sigma)$ that

$$
\nu(\sigma) \leq \max (\delta, \nu(\sigma)-(4 \sigma-2) \delta) .
$$

However, $(4 \sigma-2) \delta>0$, so that we must have $\nu(\sigma) \leq \delta$. Finally, we conclude that $\nu(\sigma) \leq 0$, since $\delta$ may be taken arbitrarily small. This proves the first part of Theorem 2 , in the case $1 / 2<\sigma \leq 1$.

To handle the case $\sigma=1 / 2$ we take $\alpha=1 / 2-\varepsilon$ in (27). We have just established that $\nu(\beta) \leq 0$ whenever $1 / 2<\beta \leq 1$, so that, in particular, $\nu(1-\alpha) \leq 0$. Thus $(27)$ yields

$$
S(Q, s) \ll(Q T U)^{\varepsilon}(Q+U)+U^{-2 \varepsilon}\left(Q+(Q T)^{1-2 \varepsilon}\right)(Q T)^{5 \varepsilon},
$$

uniformly in $t$, for $\sigma=1 / 2$. Hence, on setting $U=Q T$, for example, and taking $\varepsilon$ arbitrarily small, we see that $\nu(1 / 2) \leq 0$ also. This proves the first part of Theorem 2 in the remaining case $\sigma=1 / 2$.

To handle the remaining assertion of Theorem 2 we return to the formula (26) and separate off the sum

$$
\Sigma(s, \chi)=\sum_{n=1}^{M} d(n) \chi(n) n^{-s} e^{-n / U},
$$

where $M$ is a parameter to be chosen in due course. Proceeding as before, with $\alpha=1 / 2$ and $U=Q T$, we find that

$$
\begin{aligned}
& \sum_{\chi \in S(2 Q)-S(Q)}\left|L(s, \chi)^{2}-\Sigma(s, \chi)\right|^{2} \\
& \ll(Q T)^{3 \varepsilon}\left(Q M^{1-2 \sigma}+(Q T)^{2-2 \sigma}\right)=o(Q),
\end{aligned}
$$

for $|t| \leq Q^{(2 \sigma-1) /(2-2 \sigma)-\delta}$, providing that $M$ is at least a fixed power of $Q$.

In order to handle the sum

$$
\sum_{\chi \in S(2 Q)-S(Q)}|\Sigma(s, \chi)|^{2}
$$


we shall require the estimate

$$
\sum_{\chi \in S(2 Q)-S(Q)} \chi(n) \ll(n Q)^{1 / 2} \log n,
$$

which is valid for any positive, non-square integer $n$. We shall prove this at the end of this section. If we expand (29) to give

$$
\sum_{m, n=1}^{M} d(m) d(n) m^{-s} n^{-\bar{s}} e^{-(m+n) / U} \sum_{\chi \in S(2 Q)-S(Q)} \chi(m n),
$$

and apply (30) to those terms for which $m n$ is not a square, the corresponding contribution will be

$$
\ll Q^{1 / 2} \sum_{m, n=1}^{M} 1 \ll Q^{1 / 2} M^{2},
$$

in view of the fact that $\sigma>1 / 2$. As we shall see later, we have

$$
\sum_{\substack{q \leq Q \\(q, k)=1}}^{*} 1=Q \prod_{p \mid 2 k}\left(1-p^{-1}\right) \prod_{p \nmid 2 k}\left(1-p^{-2}\right)+O\left(Q^{1 / 2} d(k)\right) .
$$

Since the conductor of $\chi \in S(Q)$ is either an odd square-free integer, or 4 times such an integer, or (in two cases) 8 times such an integer, it follows that

$$
\begin{aligned}
\sum_{\chi \in S(2 Q)-S(Q)} \chi\left(k^{2}\right) & =Q \prod_{p \mid k}\left(1-p^{-1}\right) \prod_{p \nmid k}\left(1-p^{-2}\right)+O\left(Q^{1 / 2} d(k)\right) \\
& =Q \frac{6}{\pi^{2}} \prod_{p \mid k}\left(1+p^{-1}\right)^{-1}+O\left(Q^{1 / 2} d(k)\right)
\end{aligned}
$$

if $k$ is even, and

$$
\begin{aligned}
\sum_{\chi \in S(2 Q)-S(Q)} \chi\left(k^{2}\right) & =\frac{3}{2} Q \prod_{p \mid 2 k}\left(1-p^{-1}\right) \prod_{p \nmid 2 k}\left(1-p^{-2}\right)+O\left(Q^{1 / 2} d(k)\right) \\
& =Q \frac{9}{\pi^{2}} \prod_{p \mid 2 k}\left(1+p^{-1}\right)^{-1}+O\left(Q^{1 / 2} d(k)\right)
\end{aligned}
$$

if $k$ is odd. We therefore see that, in either case,

$$
\sum_{\chi \in S(2 Q)-S(Q)} \chi\left(k^{2}\right)=\frac{6}{\pi^{2}} F(k) Q+O\left(Q^{1 / 2} d(k)\right),
$$

where $F(k)$ is the multiplicative function satisfying $F\left(p^{e}\right)=p /(p+1)$ for every prime $p$. 
We now see that the contribution to (29) corresponding to terms for which $m n$ is a square will be

$$
\frac{6}{\pi^{2}} Q \sum_{m, n \leq M, m n=\square} d(m) d(n) m^{-s} n^{-\bar{s}} e^{-(m+n) / U} F(m n)+O\left(Q^{1 / 2} M^{2}\right),
$$

on observing, as before, that $\sigma$ is strictly greater than one-half. Since $U=$ $Q T$, we may remove the factor $e^{-(m+n) / U}=1+O(M / Q)$ at the expense of a total error $O\left(M^{2}\right)$, and then extend the sum so as to run over all pairs $m, n$ with $m n=\square$, at the cost of a further error

$$
\ll Q \sum_{m n=\square, m n>M}(m n)^{\varepsilon-\sigma} \ll Q \sum_{k>M^{1 / 2}} k^{3 \varepsilon-2 \sigma} \ll Q M^{3 \varepsilon-(2 \sigma-1) / 2} .
$$

On comparing all our estimates we now find that (29) is

$$
C Q+O\left(Q^{1 / 2} M^{2}\right)+O\left(Q M^{3 \varepsilon-(2 \sigma-1) / 2}\right),
$$

where the constant $C$ is given by

$$
C=\frac{6}{\pi^{2}} \sum_{m n=\square} d(m) d(n) m^{-s} n^{-\bar{s}} F(m n) .
$$

We choose $M=Q^{1 / 8}$, so that

$$
\sum_{\chi \in S(2 Q)-S(Q)}|\Sigma(s, \chi)|^{2}=C Q+o(Q) .
$$

In view of (28) we have

$$
\sum_{\chi \in S(2 Q)-S(Q)}\left|L(s, \chi)^{2}-\Sigma(s, \chi)\right| \cdot|\Sigma(s, \chi)|=o(Q),
$$

by Cauchy's inequality, and the theorem then follows, on expressing $|L(s, \chi)|^{4}$ as

$$
\left|L(s, \chi)^{2}-\Sigma(s, \chi)\right|^{2}+|\Sigma(s, \chi)|^{2}+2 \Re\left\{\left(L(s, \chi)^{2}-\Sigma(s, \chi)\right) \overline{\Sigma(s, \chi)}\right\} .
$$

It remains to establish the estimates (30) and (32). For (30) it suffices to consider

$$
\sum_{q \leq Q}^{*} \chi_{n}(q)
$$

for a non-principal character $\chi_{n}$ whose conductor is of order $n$. However, the above sum is

$$
\sum_{d \leq Q^{1 / 2}}^{\text {odd }} \mu(d) \chi_{n}\left(d^{2}\right) \sum_{k \leq Q d^{-2}}^{\text {odd }} \chi_{n}(k) \ll \sum_{d \leq Q^{1 / 2}} n^{1 / 2} \log n \ll(n Q)^{1 / 2} \log n .
$$


To prove (32) we observe that

$$
\begin{aligned}
\sum_{\substack{q \leq Q \\
(q, k)=1}}^{*} 1 & =\sum_{a \mid 2 k} \mu(a) \sum_{(b, 2 k)=1} \mu(b) \sum_{q \leq Q, a b^{2} \mid q} 1 \\
& =\sum_{a \mid 2 k} \mu(a) \sum_{(b, 2 k)=1, b \leq Q^{1 / 2}} \mu(b)\left(a^{-1} b^{-2} Q+O(1)\right) \\
& =Q \prod_{p \mid 2 k}\left(1-p^{-1}\right)\left\{\sum_{(b, 2 k)=1, b \leq Q^{1 / 2}} \mu(b) b^{-2}\right\}+O\left(Q^{1 / 2} d(k)\right) \\
& =Q \prod_{p \mid 2 k}\left(1-p^{-1}\right)\left\{\prod_{p \nmid 2 k}\left(1-p^{-2}\right)+O\left(Q^{-1 / 2}\right)\right\}+O\left(Q^{1 / 2} d(k)\right) \\
& =Q \prod_{p \mid 2 k}\left(1-p^{-1}\right) \prod_{p \nmid 2 k}\left(1-p^{-2}\right)+O\left(Q^{1 / 2} d(k)\right)
\end{aligned}
$$

as required.

11. Proof of Theorem 3. For the proof of Theorem 3 it will suffice to show that the number $N$ of characters $\chi \in S(Q)$ for which $L(s, \chi)$ has a zero in the square

$$
\sigma \leq \Re(s)<\sigma+(\log Q T)^{-1}, \quad \tau \leq \Im(s)<\tau+(\log Q T)^{-1}
$$

satisfies

$$
N \ll(Q T)^{\varepsilon}\left(Q^{3} T\right)^{(1-\sigma) /(2-\sigma)}
$$

for $|\tau| \leq T$. We assume that $\sigma>1 / 2+(\log Q T)^{-1}$, since otherwise the required bound is trivial. Following the standard procedure laid down by Montgomery [6; Chapter 12], we give ourselves parameters $Y \gg X \gg 1$, and define a function

$$
M_{X}(s, \chi)=\sum_{n \leq X} \mu(n) \chi(n) n^{-s} .
$$

Two cases then arise. Firstly, there may be $\gg N$ characters $\chi$, with corresponding zeros $\varrho=\beta+i \gamma$, for which

$$
\left|\int_{-A \log Q T}^{A \log Q T} L\left(\frac{1}{2}+i \gamma+i u, \chi\right) M_{X}\left(\frac{1}{2}+i \gamma+i u, \chi\right) Y^{1 / 2-\beta+i u} \Gamma\left(\frac{1}{2}-\beta+i u\right) d u\right| \gg 1 .
$$

Secondly, there may be an integer $U$ in the range $X \leq U \leq Y^{2}$, and $\gg$ $N(\log Y)^{-1}$ characters $\chi$, with corresponding zeros $\varrho=\beta+i \gamma$, for which

$$
\left|\sum_{U<n \leq 2 U} a_{n} \chi(n) n^{-\varrho} e^{-n / Y}\right| \gg(\log Y)^{-1} .
$$


Here $a_{n}=a_{n}(X)$ is given by the expansion

$$
L(s, \chi) M_{X}(s, \chi)=\sum_{n=1}^{\infty} a_{n} \chi(n) n^{-s} .
$$

In the first case, since $\varrho$ must lie in the square (34) we see that

$$
\int_{\tau-A \log Q T}^{\tau+1+A \log Q T}\left|L\left(\frac{1}{2}+i u, \chi\right) M_{X}\left(\frac{1}{2}+i u, \chi\right)\right| d u \gg Y^{\sigma-1 / 2}(\log Q T)^{-1} .
$$

We now apply Hölder's inequality, and sum over the characters, to obtain the bound

$$
\begin{aligned}
N\left(Y^{\sigma-1 / 2}(\log Q T)^{-1}\right)^{4 / 3}(\log Q T)^{-1 / 3} & \\
\ll & \int_{\tau-A \log Q T}^{\tau+1+A \log Q T} \sum_{\chi \in S(Q)}\left|L\left(\frac{1}{2}+i u, \chi\right) M_{X}\left(\frac{1}{2}+i u, \chi\right)\right|^{4 / 3} d u \\
\ll & \left(\int_{\tau-A \log Q T}^{\tau+1+A \log Q T} \sum_{\chi \in S(Q)}\left|L\left(\frac{1}{2}+i u, \chi\right)\right|^{4} d u\right)^{1 / 3} \\
& \times\left(\int_{\tau-A \log Q T}^{\tau+1+A \log Q T} \sum_{\chi \in S(Q)}\left|M_{X}\left(\frac{1}{2}+i u, \chi\right)\right|^{2} d u\right)^{2 / 3} .
\end{aligned}
$$

The first integral will be $O\left((Q T)^{1+\varepsilon}\right)$, by Theorem 2 , and the second integral may be handled by breaking the sum for $M_{X}(s, \chi)$ into ranges $V<n \leq 2 V$ and applying Cauchy's inequality. Thus

$$
\begin{aligned}
& \int_{\tau-A \log Q T}^{\tau+1+A \log Q T} \sum_{\chi \in S(Q)}\left|M_{X}\left(\frac{1}{2}+i u, \chi\right)\right|^{2} d u \\
& \ll(\log X) \sum_{V} \int_{\tau-A \log Q T} \sum_{\chi \in S(Q)}\left|\sum_{V<n \leq 2 V} \mu(n) \chi(n) n^{-1 / 2-i u}\right|^{2} d u \\
& \ll(\log X) \sum_{V}(Q V)^{\varepsilon}(Q+V) \\
& \ll(Q X)^{2 \varepsilon}(Q+X),
\end{aligned}
$$

by Corollary 3 . We therefore obtain

$$
N \ll Y^{(2-4 \sigma) / 3}(Q T)^{1 / 3}(Q+X)^{2 / 3}(Q T Y)^{3 \varepsilon},
$$

in the first case.

We now examine the second case. We shall assume that $Y \leq(Q T)^{A}$ for some constant $A$. If $\varrho$ is in the square (34) we see via partial summation 
that

$$
\begin{aligned}
& \sum_{U<n \leq 2 U} a_{n} \chi(n) n^{-\varrho} e^{-n / Y} \\
& \ll\left|\sum_{U<n \leq 2 U} a_{n} \chi(n) n^{-s} e^{-n / Y}\right|+\int_{U}^{2 U}\left|\sum_{U<n \leq V} a_{n} \chi(n) n^{-s} e^{-n / Y}\right| \frac{d V}{V},
\end{aligned}
$$

where $s=\sigma+i \tau$. It follows from Cauchy's inequality that

$$
\begin{array}{r}
\left|\sum_{U<n \leq 2 U} a_{n} \chi(n) n^{-s} e^{-n / Y}\right|^{2}+\int_{U}^{2 U}\left|\sum_{U<n \leq V} a_{n} \chi(n) n^{-s} e^{-n / Y}\right|^{2} \frac{d V}{V} \\
\gg(\log Q T)^{-2}
\end{array}
$$

for $\gg N(\log Q T)^{-1}$ characters $\chi$. Thus, on summing over all $\chi \in S(Q)$ we have

$$
\begin{aligned}
\sum_{\chi \in S(Q)}\left|\sum_{U<n \leq 2 U} a_{n} \chi(n) n^{-s} e^{-n / Y}\right|^{2} & \\
& +\int_{U}^{2 U} \sum_{\chi \in S(Q)}\left|\sum_{U<n \leq V} a_{n} \chi(n) n^{-s} e^{-n / Y}\right|^{2} \frac{d V}{V} \\
\gg & N(\log Q T)^{-3} .
\end{aligned}
$$

However, Corollary 3 shows that

$$
\sum_{\chi \in S(Q)}\left|\sum_{U<n \leq V} a_{n} \chi(n) n^{-s} e^{-n / Y}\right|^{2} \ll(Q T)^{\varepsilon}(Q+U) U^{1-2 \sigma} e^{-U / Y},
$$

since $\left|a_{n}\right| \leq d(n) \ll n^{\varepsilon}$ for any $\varepsilon>0$. We therefore conclude in the second case that

$$
N \ll(Q T)^{2 \varepsilon}(Q+U) U^{1-2 \sigma} e^{-U / Y},
$$

for some $U$ in the range $X \leq U \leq Y^{2}$, and hence that

$$
N \ll(Q T)^{2 \varepsilon}\left(Q X^{1-2 \sigma}+Y^{2-2 \sigma}\right) .
$$

A comparison of (36) and (37) now shows that

$$
N \ll(Q T)^{\varepsilon}\left\{Y^{(2-4 \sigma) / 3}(Q T)^{1 / 3}(Q+X)^{2 / 3}+Q X^{1-2 \sigma}+Y^{2-2 \sigma}\right\}
$$

for any $\varepsilon>0$. It remains to choose $X$ and $Y$ optimally. This may be done by taking $X=Q$ and

$$
Y=\left(Q^{3} T\right)^{1 /(4-2 \sigma)},
$$

whence

$$
N \ll(Q T)^{\varepsilon}\left(Q^{3} T\right)^{(1-\sigma) /(2-\sigma)} .
$$

This establishes the bound (35) and Theorem 3 follows. 
Acknowledgement. It is a pleasure to record the support of the Isaac Newton Institute, Cambridge, where much of this work was carried out.

\section{References}

[1] P. D. T. A. Elliott, On the mean value of $f(p)$, Proc. London Math. Soc. (3) 21 (1970), 28-96.

[2] P. X. Gallagher, The large sieve, Mathematika 14 (1967), 14-20.

[3] M. Jutila, On mean values of L-functions and short character sums with real characters, Acta Arith. 26 (1975), 405-410.

[4] -, On mean values of Dirichlet polynomials with real characters, ibid. 27 (1975), 191-198.

[5] -, On the mean value of $L\left(\frac{1}{2}, \chi\right)$ for real characters, Analysis 1 (1981), 149-161.

[6] H. L. Montgomery, Topics in Multiplicative Number Theory, Lecture Notes in Math. 227, Springer, Berlin, 1971.

MAGDALEN COLLEGE

OXFORD OX14AU, ENGLAND 